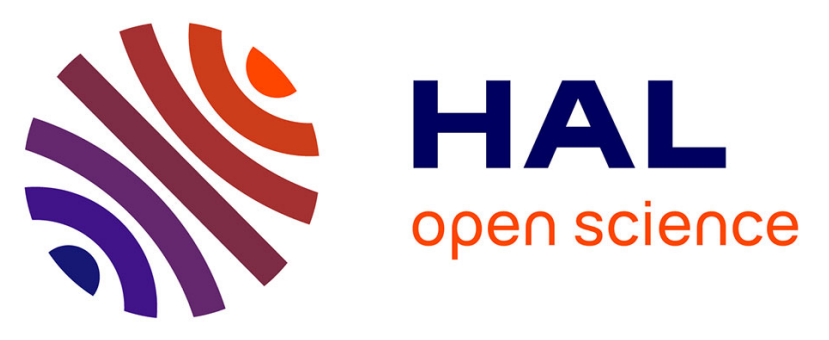

\title{
Terahertz multi-plasmon induced reflection and transmission and sensor devices in a graphene-based coupled nanoribbons resonators
}

Adnane Noual, Madiha Amrani, El Houssaine El Boudouti, Yan Pennec, Bahram Djafari-Rouhani

\section{To cite this version:}

Adnane Noual, Madiha Amrani, El Houssaine El Boudouti, Yan Pennec, Bahram DjafariRouhani. Terahertz multi-plasmon induced reflection and transmission and sensor devices in a graphene-based coupled nanoribbons resonators. Optics Communications, 2019, 440, pp.1-13. 10.1016/j.optcom.2019.02.002 . hal-03140656

\section{HAL Id: hal-03140656 https://hal.science/hal-03140656}

Submitted on 18 Aug 2021

HAL is a multi-disciplinary open access archive for the deposit and dissemination of scientific research documents, whether they are published or not. The documents may come from teaching and research institutions in France or abroad, or from public or private research centers.
L'archive ouverte pluridisciplinaire HAL, est destinée au dépôt et à la diffusion de documents scientifiques de niveau recherche, publiés ou non, émanant des établissements d'enseignement et de recherche français ou étrangers, des laboratoires publics ou privés.

\section{(c)(1)}

Distributed under a Creative Commons Attribution| 4.0 International License 


\title{
Terahertz multi-plasmon induced reflection and transmission and sensor devices in a graphene-based coupled nanoribbons resonators
}

\author{
A. Noual ${ }^{\text {a,b,*, M. Amrani }}{ }^{\text {a }}$, E.H. El Boudouti ${ }^{\text {a }}$, Y. Pennec $^{c}$, B. Djafari-Rouhani ${ }^{c}$ \\ a LPMR, Département de Physique, Faculté des Sciences, Université Mohammed Premier, 60000 Oujda, Morocco \\ ${ }^{\mathrm{b}}$ Département de Physique, Faculté Pluridisciplinaire Nador, Université Mohammed Premier, 60000 Oujda, Morocco \\ ${ }^{\mathrm{c}}$ IEMN, UMR CNRS 8520, Département de Physique, Université de Lille, 59655 Villeneuve d'Ascq, France
}

\begin{abstract}
We demonstrate numerically multi-plasmon-induced reflection (PIR), multi-plasmon-induced transparency (PIT) and propose a highly sensitive refractive index nanosensor in a novel ultra-compact graphene-based nano-device, operating in the terahertz frequency range. The base structure of the system is composed out of a bus waveguide made out of graphene coupled to resonators inserted along or aside the waveguide where each resonator is constituted by a set of two coupled graphene nano-ribbons (CGNR). The latters behave as a molecule-like entity, whose surface plasmon eigen-modes originate from the splitting of the single ribbons modes. We show that the two CGNRs can also be regarded as an effective rectangular cavity-like system, so that the insertion of the latter along or aside the graphene bus waveguide (GBW) enables the design of tunable selective or rejective filters, respectively. By inserting two identical effective cavities in the structure, into a $\Lambda$ like state (in analogy with three level atomic systems), we show the possibility of realizing multiple plasmon induced reflection, and highlight its fast light-features. On the other hand, if the two identical cavities are set symmetrically on each side of the GBW with slightly detuned Fermi energy levels in a V-like configuration, multi-plasmon induced transparency can be obtained. In addition, a highly sensitive refractive index nanosensor is showcased. The latter consists in a GBW, sidecoupled to an effective cavity-oscillator embedded with the analyte to be detected. The sensitivity of the sensor is numerically derived. Owing to its simple structure, the proposed sensor may offer the advantage of being easily fabricated. The showcased graphene-based devices proposed in this work should help the design of tunable and highly integrated optical devices such as nano-filters, optical switches and highly sensitive nanosensors.
\end{abstract}

\section{Introduction}

Electromagnetically induced transparency (EIT) is a well-known physical effect in atomic systems, that arises because of quantum destructive interferences between two excitation pathways to an upper atomic level [1]. Steep dispersion and low absorption take place in a sharp transparency window, which makes it very attractive for a plenty of potential applications in slowing light, enhancing optical nonlinearity and data storage [2-4]. Nevertheless, the realization of EIT is greatly hindered owing to the extreme required conditions at the experimental level [5]. To overcome these difficulties, a great deal of attention has been paid to mimic the EIT-effect in classical optical systems $[6,7]$. Specifically, the plasmon-induced transparency (PIT) has sparked tremendous efforts due to the unique ability of surface plasmonpolaritons (SPPs) to confine and manipulate light waves below the classical diffraction limit [8,9]. Plasmonic devices for PIT are usually based on the metal-insulator-metal (MIM) waveguide structure; this has been shown both theoretically and experimentally in Refs. [10,11] for instance. However, MIM structures are built based on noble metals, and it is quite hard to adjust the noble metal materials permittivity, which renders their utilization in some plasmonic components rather limited. On the other side, graphene is a carbon atom thick layer with astonishing and unique optical and physical properties. Surface plasmon excitation on a graphene layer has been shown [12] and, as compared to surface plasmon propagation in metals, graphene plasmons manifest a higher confinement and relatively low propagation loss [13]. One of the key advantages of graphene over noble metals is the possibility to dynamically tune its conductivity by changing its Fermi-level energy; this is performed in practice by applying a voltage gate on graphene layer [14]. A merge between graphene physics and plasmonics has been introduced lately, to design graphene-based meta-surfaces [15,16], optical waveguides $[17,18]$, switches [19,20], photo-detectors [21,

* Correspondence to: Laboratoire de Physique de la Matière et de Rayonnements, Département de Physique, Faculté Pluridisciplinaire Nador, Université Mohammed Premier, 60000 Oujda, Morocco.

E-mail address: noualad@yahoo.fr (A. Noual). 


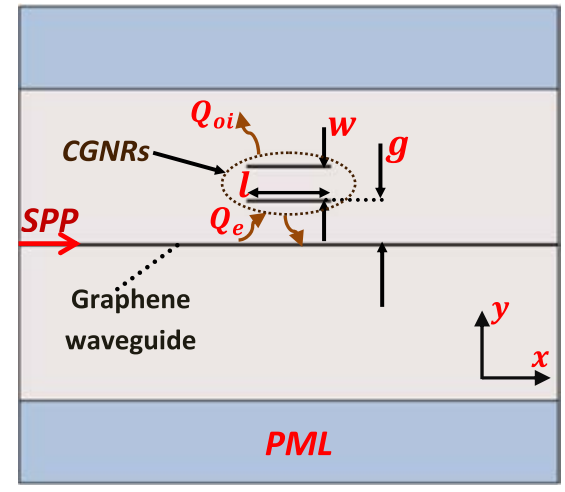

Fig. 1. Schematic diagram of a graphene-based waveguide structure coupled to two CGNRs, placed aside of the waveguide.

22], filters [23,24], refractive index sensors [25,26] and directionalcoupler [27]. Moreover, PIT-effect has been shown numerically and theoretically in a graphene-based Fabry-Perot microcavity [28]. In graphene-based waveguides structures, tunable PIT-effects have been realized [29-31]; this should certainly benefit on-chip plasmonic devices.

Plasmon-induced reflection (PIR) is a similar phenomenon to PIT as it arises from extreme destructive interferences between the dark mode and the bright mode [32-34]. Contrary to PIT, it generates a reflection window in the transparency band of the system. Lately, PIR-phenomenon has been investigated theoretically $[35,36]$ and experimentally [37] in metamaterials made of wire-slot and split-ring resonator arrays. Furthermore, authors in Ref. [38] demonstrated the possibility of harvesting PIR-resonances in order to realize a narrowband perfect absorber with two absorption peaks to conceive a plasmonic sensor. Also, Liu et al. have shown that PIR-effects can be utilized in planar metamaterials for plasmonic sensing applications [39]. In some other recent papers dealing with graphene-based waveguide structures [40-42], the existence of dips in the transmission spectrum is referred to as plasmon-induced absorption (PIA). However, the reflection and absorption spectra are not independently analysed in these works and a distinction between PIR and PIA becomes useful. Indeed, within PIR-frequency window the reflection spectrum peaks-up while, the absorption coefficient decreases or is not affected. Within a PIA window, the absorption coefficient should peak-up (or get enhanced) as this is clearly shown in some metamaterials-based structures. Let us point out that due to its abnormal dispersion and unique fast-light feature, PIR-effect, has a great value with many potential applications such as ultrafast optical switching [43] and fast light applications [44]. In particular, as opposed to PIT/PIA phenomena, PIR effect has been less investigated in the literature, especially in graphene-based nanostructures. In that spirit, and motivated by all the above studies, in this paper, we propose and analyse the functionalities and performances of a novel graphene-based ultra-compact nanoscale structure for the realization of multi-PIR effect, operating in the terahertz frequency range. Also, we show that multi-PIT resonances and a highly sensitive nano-sensor can be achieved based on the studied structure.

The base geometry design of the structure is composed out of a graphene bus waveguide (GBW) and two coupled identical graphene nano-ribbons (CGNRs). The latters act as a molecule-like system whose eigen surface plasmon-polariton (SPP) modes arise from the strong coupling between the ribbons and play the role of an effective (rectangular) cavity-resonator. As a matter of fact, this is one of the key advantages of the proposed structure, given the simplicity and compactness of the two CGNRs as opposed to the different (graphene-based) resonators geometries usually showcased in the literature to realize PIT/PIR phenomena [40]. More specifically, the simplicity of our (effective) resonator might reduce additional (and possibly challenging) fabrication steps for experimental realizations. By placing the effective cavity along or aside of the GBW one produces multi-selective or rejective filters, which can be dynamically tuned by shifting the Fermi energy levels of the ribbons. Multi-PIR effect is generated by inserting two identical effective cavities in the structure, such that one of the latters is placed along the GBW, and the other one vertically above in a $\Lambda$-like state (in analogy with the three level atomic systems). On the other hand, multi-PIT resonances are achieved by slightly detuning the Fermi energy level of the two identical cavities inserted on each side of the GBW (in a V-like state). Finally, we show the possibility of realizing a highly sensitive refractive index sensor, by inserting one cavity oscillator aside of the GBW and filling it with the material to be studied (for instance a gas or a liquid). Given its simplicity and versatility, the proposed structure may play a significant role in realizing ultra-compact, nano-integrated devices and circuits in the $\mathrm{THz}$ range for optical communication and or nano-sensing.

This paper is organized as follows: in Section 2, theoretical and numerical approaches used in this study are detailed. In Section 3, we present the results and discussions about multi-PIR and PIT resonances and the performance of the nano-sensor. The conclusion is given in Section 4.

\section{Theory and numerical methods of simulation}

A two dimensional sketch of one of the base structures of our proposed device is presented in Fig. 1, which is a multi-mode rejective filter. In that configuration, the two CGNRs are placed aside in the upper half space of the system at a distance $g$ (counting from the lower ribbon) from the GBW. The two CGNRs may be regarded as an effective rectangular cavity-like oscillator, characterized by its length $l$ (ribbons length) and width $w$ (separation between the ribbons). The latter reflects to the coupling intensity between the ribbons. For practical applications, the graphene-based nanostructure must be embedded in a dielectric such as $\mathrm{SiO}_{2}$ [45]. Following that, the proposed device is placed in a dielectric medium with a dielectric permittivity of $\varepsilon=2.25$. The in-plane cross section of the structure ( $x y$-plane) is investigated and analysed based on Finite Element Method. Specifically, the versatile software Comsol-Multiphysics package has been used to perform the numerical simulations. In our model we treat graphene as a very thin anisotropic material with a thickness $\Delta$, which we chose throughout the paper to be equal to $1 \mathrm{~nm}$ [46]. The effective surface permittivity of graphene is given by $\varepsilon_{s}=1+\sigma / \varepsilon_{0} \omega \Delta$ [47], whereas its normal permittivity is $\varepsilon_{\perp}=2.5$ [48]. Here, $\varepsilon_{0}$ is the vacuum permittivity, $\omega$ is the light angular frequency and $\sigma$ is the surface conductivity of graphene. The latter is composed out of two contributions: interband transitions and intra-band electron-photon scattering based on the Kubo formula [46]. In the terahertz frequency region, the photon energy is always smaller than $2 E_{F}$ (where $E_{F}$ is the Fermi energy level) [49], and consequently the intra-band contribution dominates the inter-band contribution. As a result, $\sigma$ simplifies to a Drude-like expression, that is, $\sigma=i e^{2} E_{F} / \pi \hbar^{2}\left(\omega+i \tau^{-1}\right)$ [50], where $e, \hbar, \tau$ are the electron charge, the reduced Planck constant and the relaxation time, respectively. The carrier relaxation time is related to the Fermi velocity, $v_{F}=10^{6} \mathrm{~m} / \mathrm{s}$ [51], and to the carrier mobility $\mu$ as $\tau=\mu E_{F} / e v_{F}^{2}$. We assumed a value of $10^{5} \mathrm{~cm}^{2} \mathrm{~V}^{-1} \mathrm{~s}^{-1}$ for $\mu$ as in [51].

The Fermi energy level of the two CGNRs is identical and is referred to as $E_{F 1}$, whilst $E_{F 0}$ corresponds to the GBW Fermi energy level. We set a fixed value for $E_{F 0}$ in this work such as $E_{F 0}=140 \mathrm{meV}$. A TMpolarized SPP-eigen mode of the GBW is excited and launched from the left port; the transmission spectrum is then recorded at the right end of the waveguide. In order to get rid of any possible reflections perturbing the system, we put up PML layers on the bottom and upper sides of the structure (Fig. 1). It is worth indicating that the SPPs propagation constant supported by a graphene layer can be easily derived as [49]:

$\beta_{S P P}=k_{0} \cdot n_{e f f}=k_{0} \sqrt{1-\left(\frac{2}{\eta_{0} \sigma}\right)^{2}}$ 
Here, $n_{\text {eff }}$ is the effective refractive index of SPPs supported by graphene, $k_{0}$ is the wave-vector in vacuum and $\eta_{0}$ is the air impedance. Based on this formula, the SPPs properties can be tuned by adjusting the Fermi energy level (proportional to $\sigma$ ) via an external gate-voltage.

\section{Results and discussion}

\subsection{Single cavity side-coupled to the waveguide}

Before studying multi-PIR and PIT effects, we first investigate the plasmonic properties of a single cavity-resonator side-coupled with the main waveguide (Fig. 1). Specifically, a fundamental study of the different cavity modes and their behaviour versus the geometrical parameters is performed. This study enables us to choose appropriately the cavity dimensions in order to realize the multi-PIR/PIT phenomenon. Afterwards, the transmission properties of the system when the cavity is inserted along the waveguide are examined (see Fig. 5(a) and Section 3.2). The latter configuration of the system constitutes the building block of the typical geometry design giving rise to PIR.

Fig. 2(a) presents the transmission spectrum of the system depicted in Fig. 1 (red curve with red dot markers) in the case where $l=250 \mathrm{~nm}$, $w=110 \mathrm{~nm}$ and $g=130 \mathrm{~nm}$. The Fermi energy level of the two CGNRs is chosen such as $E_{F 1}=160 \mathrm{meV}$. As a matter of comparison the transmission coefficient of a single nano-ribbon (of length $l=250 \mathrm{~nm}$ and with $E_{F 1}=160 \mathrm{meV}$ ) side coupled to the GBW, at a distance $g=130 \mathrm{~nm}$ from the former is also shown (black curve with black dot markers). In the latter case, one can notice two transmission dips at around $f_{r 1}=10.68 \mathrm{THz}$ and $f_{r 2}=16.2 \mathrm{THz}$, indicating two SPP modes of the ribbon in the frequency range of interest $(4.75 \mathrm{THz}-18.25 \mathrm{THz})$. When the two CGNRs interact with the GBW, four different transmission dips show up in the transmission spectrum. These modes originating from the strong coupling of the two CGNRs, fall around $f_{r 1}=8.82 \mathrm{THz}$, $f_{r 2}=12.2 \mathrm{THz}, f_{r 3}=15.39 \mathrm{THz}$ and $f_{r 4}=16.2 \mathrm{THz}$; these modes are labelled respectively (1), (2), (3) and (4) in Fig. 2(a). To get a deeper physical insight about these modes, we have plotted in Fig. 2(b)-(e) the map of the $z$-component of the magnetic field $H_{z}$ within the structure at the four resonance frequencies. The $H_{z}$-field maps at the resonances frequencies of a single ribbon coupled to the GBW are also shown in Fig. 2(f) and (g). It can be seen from Fig. 2(b)-(e) that the incident light is trapped in between the two CGNRs, acting as an effective rectangular cavity, and reflected back to the input of the waveguide. With respect to the dotted black line (parallel to $x$-axis) at the middle of the cavity, modes (1) and (3) are symmetric, whereas modes (2) and (4) are antisymmetric.

The $H_{z}$-field maps at the transmission dips of a single ribbon in Fig. 2(f) and (g), indicate that the SPP modes of the former correspond to $m=1$ for the lower frequency mode and to $m=2$ for the higher frequency mode; where $m$ is an integer indexing the modes, along the $x$-axis. In the case of the two CGNRs forming a cavity-like system, a second integer $n$ can be added for modes indexing along the $y$-axis. In that respect, modes (1) and (2) correspond respectively to $(m=1, n=1)$ and ( $m=1, n=2)$, whilst mode (3) and (4) correspond to $(m=2, n=1)$ and ( $m=2, n=2)$, respectively. On the other hand, it can be stated that modes (1) and (2) of the two CGNRs result from the splitting of the lower frequency mode of a single ribbon, giving rise to a symmetric mode (1) and anti-symmetric mode (2), with respect to the mid-plane between the ribbons. Similarly, the high frequency mode of the ribbon splits-up into a symmetric mode (3) and anti-symmetric mode (4), with respect to the same symmetry plane. In other words, the SPPs modes of the two CGNRs observed here originate from the hybridization of surface plasmon modes of the two identical nano-ribbons due to strong near-field coupling; this is analogous to hybridized orbitals formed by two identical atoms, or similarly, to hybridized SPPs modes arising in identical metallic nanoparticles dimers [52].

It is evident that the separation distance between the interacting ribbons, $w$, defines the coupling intensity between the latters; consequently, we have investigated the modes evolution versus $w$. Moreover, the length $l$ of the ribbons can also affect the observed SPP modes of the two CGNRs. We have then calculated in Fig. 3(a) the transmission spectra of the system depicted in Fig. 1 for different values of $w$, while keeping the remaining parameters unchanged and having the same values as in Fig. 2(a). Similarly, we present the simulated transmission spectra for various values of $l$ in Fig. 3(b), the rest of the parameters being left fixed as in Fig. 2(a). According to Fig. 3(a), at $w \leq 20 \mathrm{~nm}$ (not shown), modes (1) and (2) are much less excited, while mode (4) is cut off (and no longer exists). Fig. 3(b), on the other hand shows generally that the four modes get red-shifted when the length $l$ increases. Besides, a higher order mode having the same symmetry as mode (4) according to its $H_{z}$-field map (not shown), shows up for $l \geq 270 \mathrm{~nm}$. To be more specific, we have plotted in Fig. 3(c) and (d) the resonance frequency evolution of the four modes versus $w$ and $l$, respectively. Based on Fig. 3(c), we note that modes (1) and (2) arising from the splitting of the low frequency mode of a single ribbon (referred to as (5) in Fig. 2(a)) get even further apart in terms of their resonance frequency as $w$ decreases. This is due to the fact that when $w$ decreases the coupling strength between the ribbons turns higher, which pushes the symmetric mode (1) further towards lower frequencies, whereas the anti-symmetric mode (2) is pushed towards higher frequencies. The same trend can be observed concerning modes (3) and (4), originating from the splitting of the higher frequency mode of the single ribbon (referred to as (6) in Fig. 2(a)). One can also remark that the two branches associated with modes (2) and (3) cross each other for $w=50 \mathrm{~nm}$; at this point, both modes coexist and the oscillating mode within the CGNRs is a combination of these two modes. Similar behaviours can be noticed when studying modes evolution of a classical optical cavity versus its geometrical parameters [53]. On the other hand, modes evolution versus $l$ depicted in Fig. 3(d) show globally the same trend, that is, a redshift of the four modes when $l$ increases. The redshift behaviour maybe explained knowing that the resonance wavelength of a SPP mode of a single nano-ribbon made out of graphene (embedded in a dielectric) is given by [54]:

$\lambda=\left(\frac{2 \pi \hbar c}{e}\right) \sqrt{\frac{\eta \varepsilon_{0} \varepsilon_{r} l}{E_{F}}}$

where, $l$ is the nano-ribbon length, and $\eta$ is a dimensionless fitting parameter (from the simulations data). Based on Eq. (2), the resonance wavelength is proportional to the square root of the ribbon length. We can consider that this formula still roughly holds for the two CGNRs as the SPP modes of the latters remain almost unperturbed along the $x$-axis, comparing with the modes of a single ribbon. It is also worth mentioning that mode (2) is cut off for $l \geq 310 \mathrm{~nm}$ in Fig. 3(d). Overall, the cavity modes evolution versus geometrical parameters performed in Fig. 3 indicates that one should choose $l=250 \mathrm{~nm}$ for the cavity length and $w=110 \mathrm{~nm}$ for its thickness, so that the four modes exist in the frequency range of interest and are fairly well excited. This is particularly important to obtain multi-PIR/PIT resonances.

In the light of temporal coupled mode theory (CMT), the transmission properties of the system could be fairly well captured and fitted. Indeed, if we denote by $\omega_{i}$ the resonance frequency of the effective cavity, then the complex transmission and reflection coefficients around $\omega_{i}$ are given respectively by [55]:

$$
\begin{gathered}
t_{i}=\frac{j\left(\omega_{i}-\omega\right)+\frac{\omega_{i}}{2 Q_{o i}}}{j\left(\omega_{i}-\omega\right)+\frac{\omega_{i}}{2 Q_{o i}}+\frac{\omega_{i}}{2 Q_{e i}}} \\
r_{i}=-\frac{\frac{\omega_{i}}{2 Q_{e i}}}{j\left(\omega_{i}-\omega\right)+\frac{\omega_{i}}{2 Q_{o i}}+\frac{\omega_{i}}{2 Q_{e i}}}
\end{gathered}
$$

Here, $j=\sqrt{-1}$, and $Q_{o i}$ and $Q_{e i}$ are the quality factors of the cavity associated with the frequency $\omega_{i}$, related to the intrinsic losses and to the waveguide coupling strength magnitude, respectively. The theoretical transmission spectrum (around $\omega_{i}$ ) is given by $T_{i}=\left|t_{i}\right|^{2} \times\left|t_{0}\right|^{2}$, where $\left|t_{0}\right|^{2}$ is the transmission spectrum of the bus waveguide without 


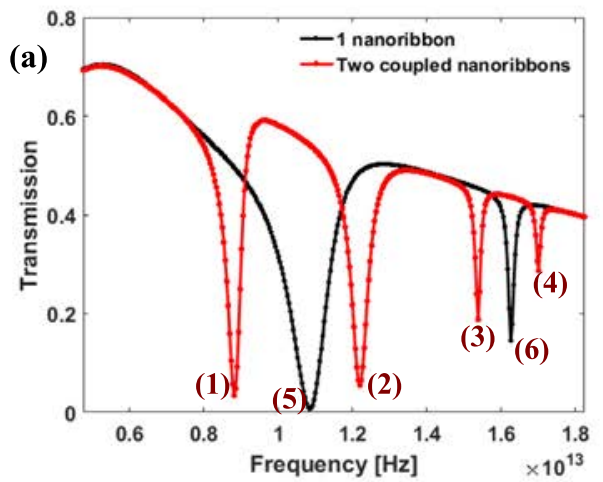

(c)

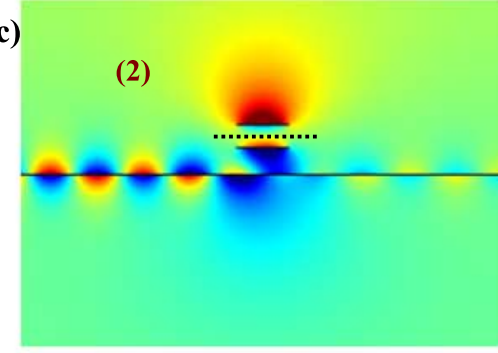

(e)

(4)

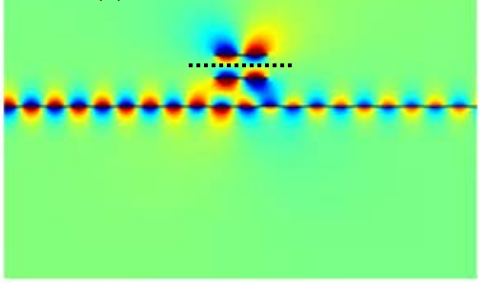

(b)

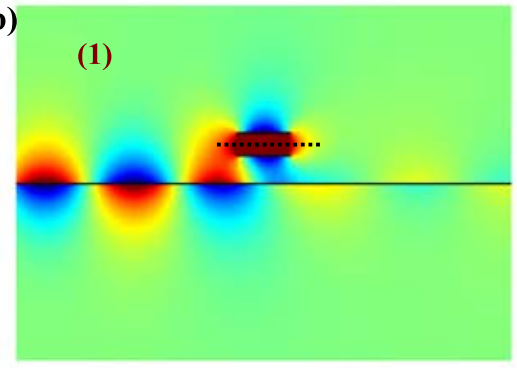

(d)

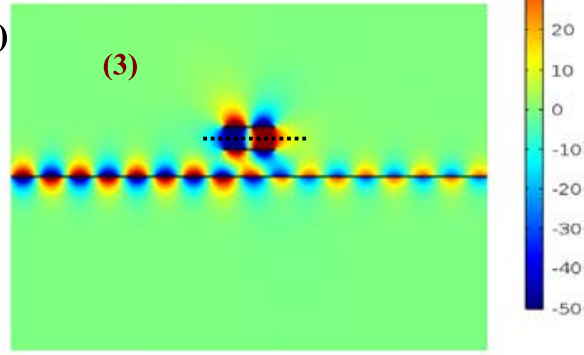

(f)

(5)

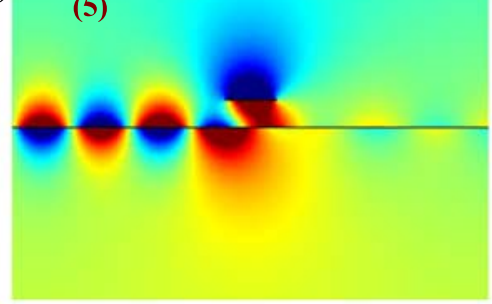

(g)

(6)

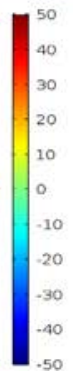

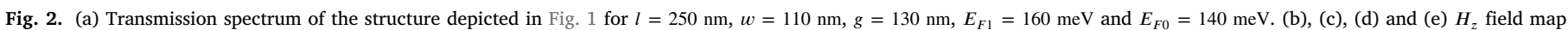

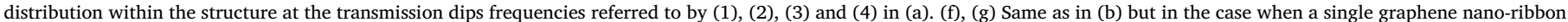
is placed aside of the GBW at a distance $g=130 \mathrm{~nm}$. (For interpretation of the references to colour in this figure legend, the reader is referred to the web version of this article.)

any cavity. The multiplication by $\left|t_{0}\right|^{2}$ enables the catching of the asymmetric profile of the main waveguide transmission spectrum as described in [55]. $Q_{e i}$ is related to the quality factor associated with the transmission zero of the resonance by $Q_{t i}=Q_{e i} \times Q_{o i} /\left(Q_{e i}+Q_{o i}\right)$, where $Q_{t i}=\omega_{i} / \Delta \omega_{i} ; \Delta \omega_{i}$ being the full width at half maximum (FWHM). $Q_{o i}$, on the other hand can be estimated using $Q_{o i}=\operatorname{real}\left(n_{e f f}\right) / 2 \operatorname{Im}\left(n_{e f f}\right)$ [56], where $n_{\text {eff }}=\beta_{\text {spp }} / k_{0}$ is the effective refractive index of the SPP mode propagating along the two CGNRs. Given that the effective cavity possesses four different resonances in the studied frequency domain, we shall follow the same method as in [57] to get the total transmission spectrum of the system associated with the four resonances. One gets:

$T=T_{1} \times T_{2} \times T_{3} \times T_{4} \times\left|t_{0}\right|^{2}$

$T_{i},(i=1,2,3$ and 4 ) being the transmission coefficient of the system associated with the resonance frequency $\omega_{i}$. In Fig. 4(a) and (b), we give two examples of the transmission spectrum of the system provided by the CMT along with the numerical one (based on FEM) for the case when $(l=250 \mathrm{~nm}, w=110 \mathrm{~nm})$ and $(l=230 \mathrm{~nm}, w=70 \mathrm{~nm})$, respectively. The remaining geometrical and physical parameters of the system (in both cases) have the same values as previously. One notes a fairly good fit of the numerical results based on CMT in both figures. Some small discrepancies (in terms of transmission values) could be observed though, between numerical and theoretical data; we attribute these to the rough approximation of $Q_{t i}$ and $Q_{e i}$. Nevertheless, both theoretical and numerical curves produce a quasi-identical profile, so that, the CMT-based fitting can significantly help understanding the numerical transmission spectra.

\subsection{Single cavity inserted along the waveguide}

In this section, we investigate the effect of inserting the effective cavity-resonator along the main waveguide (Fig. 5(a)). In this configuration, the cavity-resonator interacts with the waveguide and selects from 
(a)

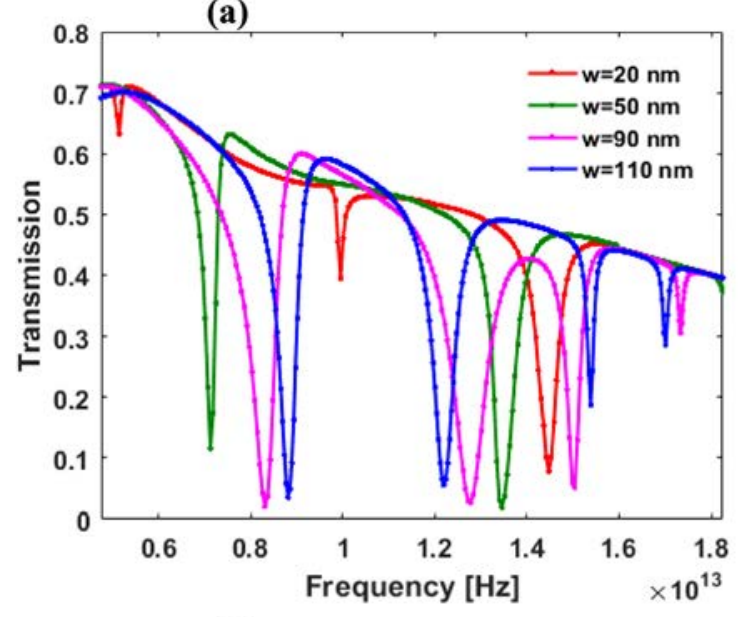

(c)

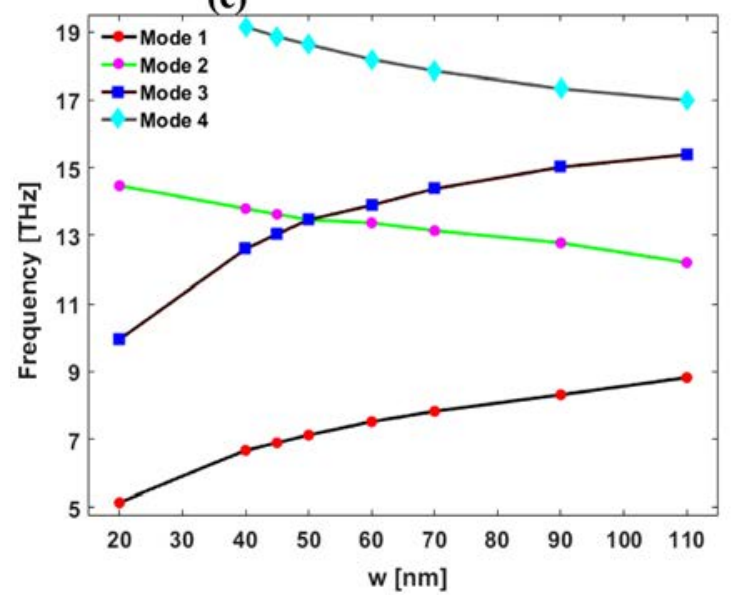

(b)

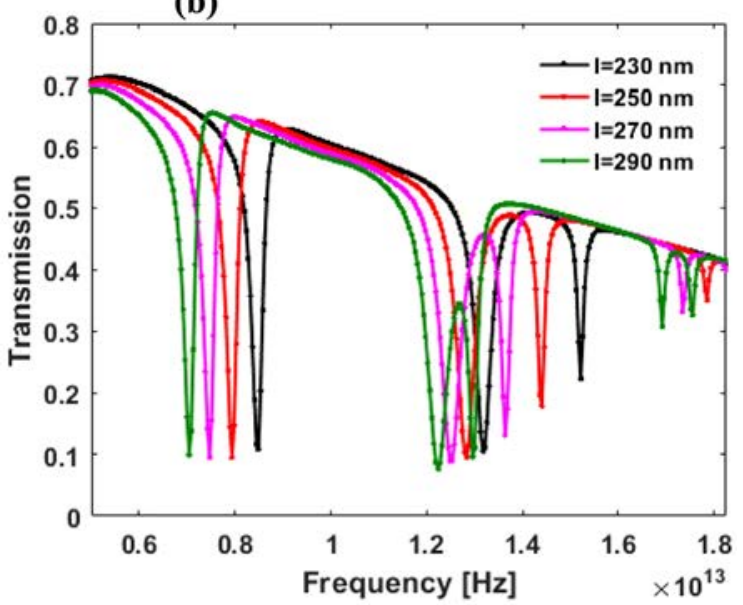

(d)

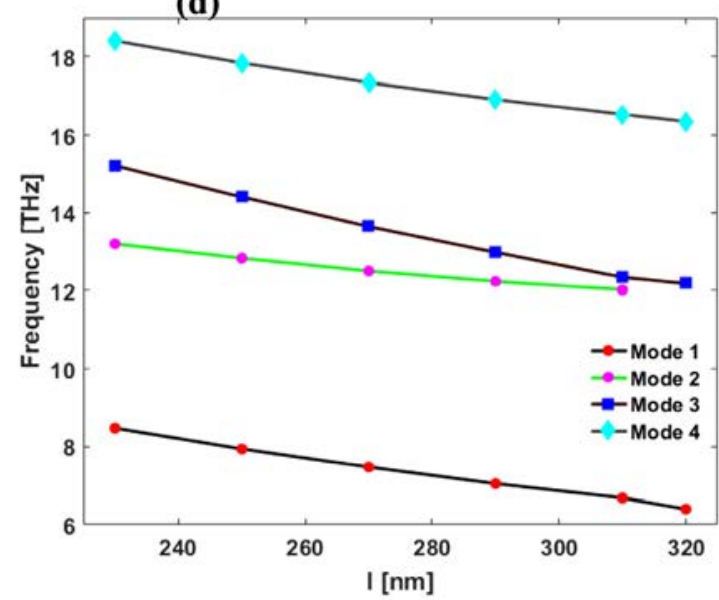

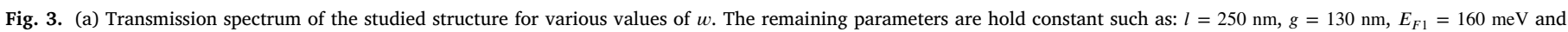

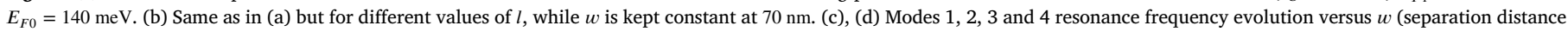
between the coupled ribbons) and $l$ (ribbons length), respectively.
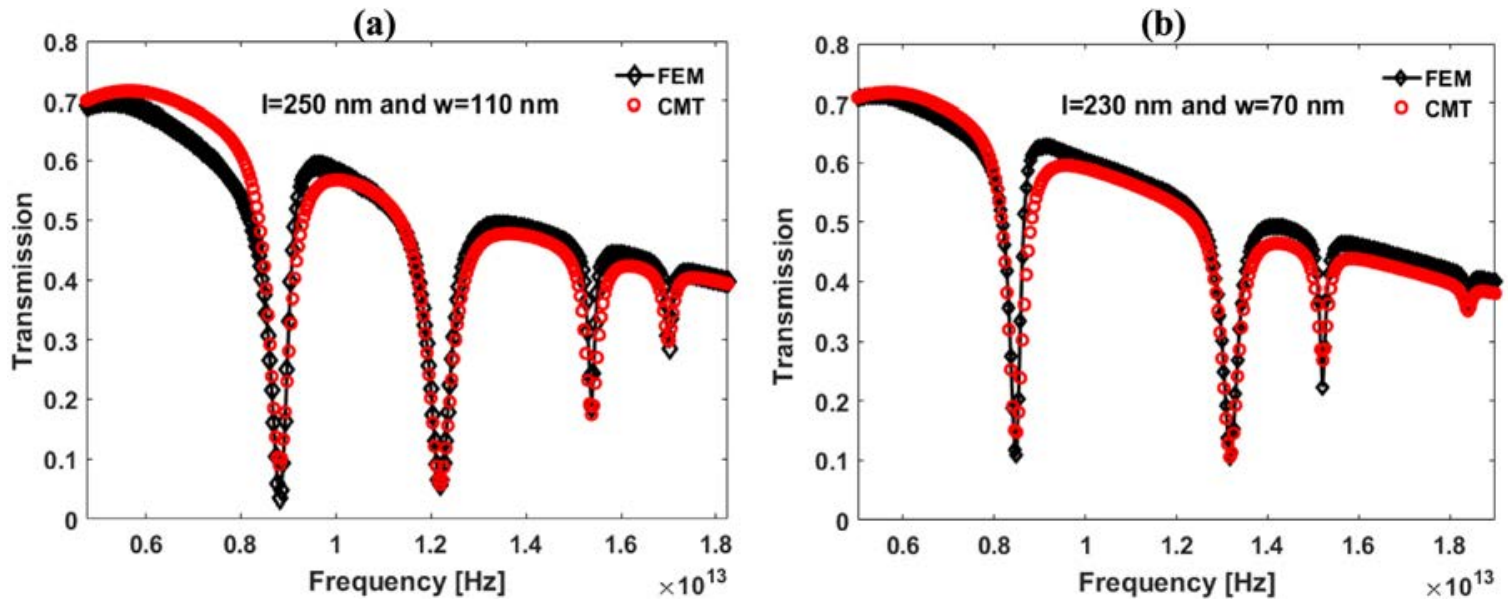

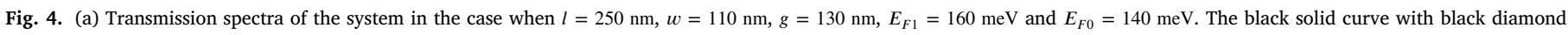

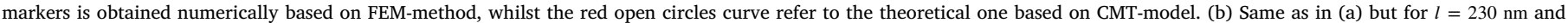
$w=70 \mathrm{~nm}$.

a large band incident signal only frequencies corresponding to its eigenSPPs modes, that is, the four modes studied above which will appear as well-defined peaks (instead of dips) in the transmission spectrum. The geometry structure is depicted in Fig. 5(a), and the corresponding transmission, reflection and absorption spectra are given in Fig. 5(b), in the case where $l=250 \mathrm{~nm}, w=110 \mathrm{~nm}, E_{F 1}=160 \mathrm{meV}, E_{F 0}=140 \mathrm{meV}$ and $d=10 \mathrm{~nm}$.

It should be mentioned that according to numerical tests (not shown here), the optimal position of the cavity which enables to get higher transmission amplitudes should be such that the lower ribbon is set exactly along the GBW (i.e., not shifted vertically with respect to the 
(a)
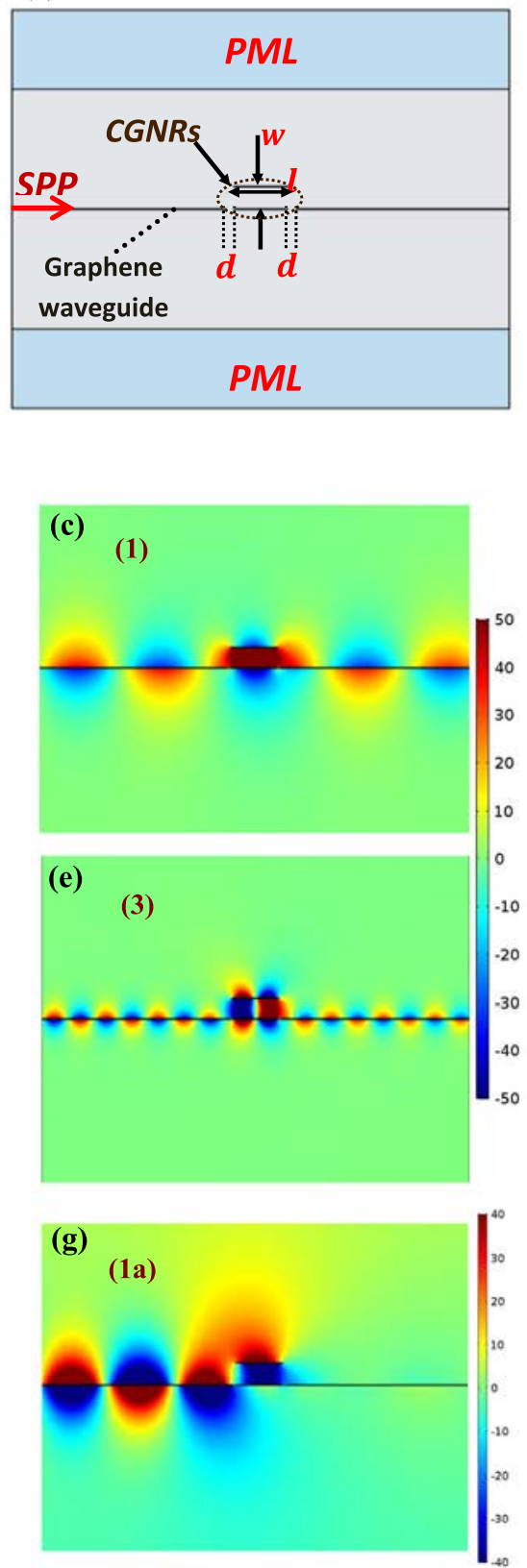

(b)
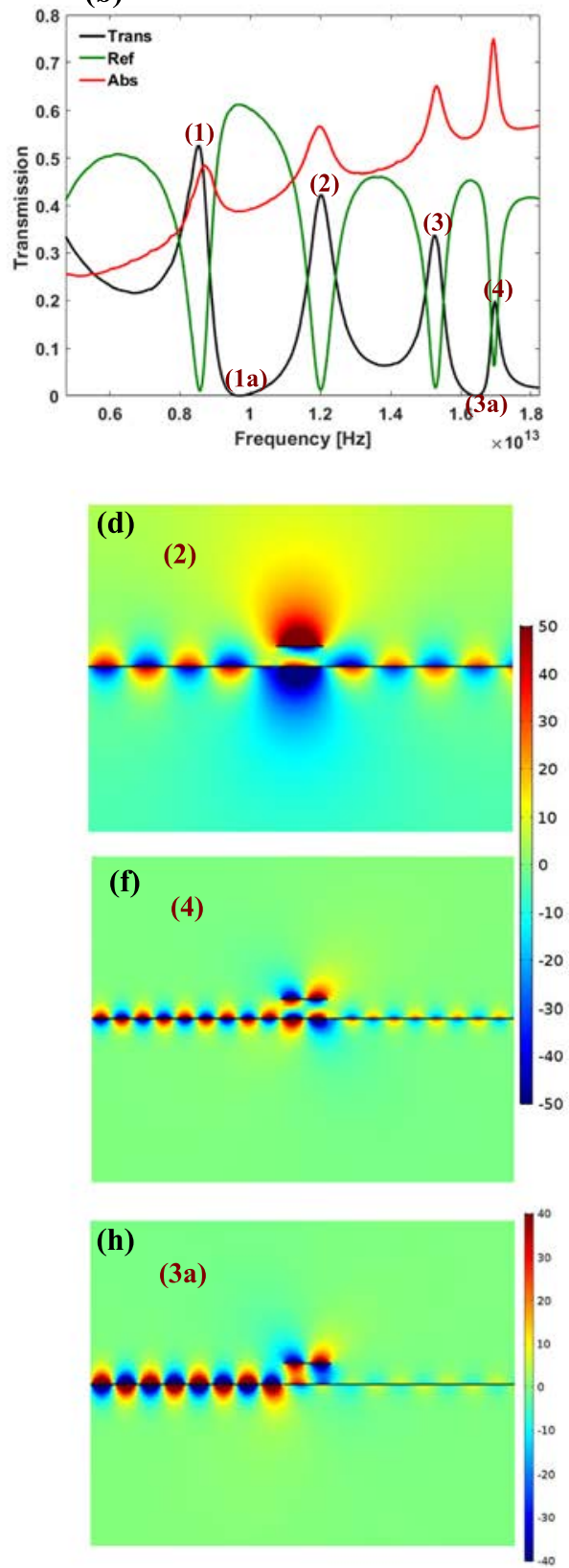

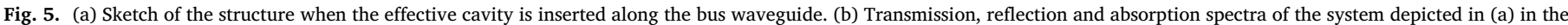

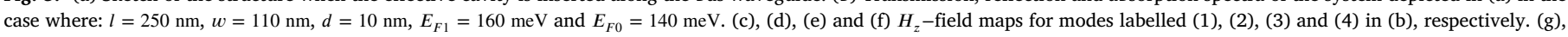
(h), $H_{z}$-field map at the transmission zeros referred to as (1a) and (3a) in (b).

main waveguide). In the same sense, the value of the parameter $d=$ $10 \mathrm{~nm}$ seems to be the optimal value based on numerical simulations, in order to get optimal transmission peaks. We notice the selective transmission of the four modes of the effective cavity, namely, the eigenSPP modes of the structure studied above which appeared as dips in (Fig. 2(a)). In the present configuration, the structure acts as a bandpass filter in contrast to the configuration in the previous section that acts as a rejection filter. Let us mention that we have already observed such behaviour [58] in a MIM-type waveguide coupled to a rectangular air-cavity put aside or along the main waveguide. When put aside, the cavity captures and reflects back towards the input the resonance frequency corresponding to one of its SPP eigen-modes, whereas when inserted along the waveguide, the cavity filters and transmits the same frequency modes towards the output system. The effective resonator studied here behaves very much in the same manner as in [58]. It should be mentioned that the filtered eigen-modes frequencies of the effective cavity are slightly different as compared to the situation where the latter is put aside. As a matter of fact, mode (1) frequency in Fig. 5(b) falls around $f_{r 1}=8.53 \mathrm{THz}$ instead of $f_{r 1}=8.82 \mathrm{THz}$ (Fig. 3(a)), this is about $3 \%$ difference. We relate this shift to the coupling strength difference between the GBW and the cavity in the two configurations. Indeed, when the resonator is along the GBW, the interaction intensity is quite higher as the guided SPP mode in this case interacts directly with the cavity. This results in a small shift of the resonance frequencies. Modes (2), (3) and (4) are now situated respectively around $f_{r 2}=12.00 \mathrm{THz}$, $f_{r 3}=15.26 \mathrm{THz}$ and $f_{r 4}=16.97 \mathrm{THz}$, which yields a percentage shift of $\approx 2 \%, 1 \%$ and $4 \%$ respectively. Fig. 5(c)-(f) give the $H_{z}$-field maps of the filtered modes labelled (1), (2), (3) and (4) in Fig. 5(b). It can be seen that the incident wave at $f_{r 1}$ (Fig. 5(c)), for instance, strongly couples with the effective cavity as the eigen-SPP mode of the resonator 
is excited; subsequently the incident wave resonantly tunnels towards the waveguide output and get transmitted. The same behaviour can be observed in Fig. 5(d)-(f). One notices that the excited eigen-SPP modes of the effective cavity (based on their structure/symmetry) are exactly the same modes as those displayed in Fig. 2(b)-(e).

On the other hand, we would like to draw attention on a wide region of low transmission occurring in-between the first and the second peaks, and between the third and fourth peaks. We refer to these transmission-like zeros as (1a) and (3a) in Fig. 5(b), respectively. The mechanism of these destructive interferences can be explained by the fact that light takes two different path ways through the lower and upper ribbons and the resulting two waves interfere destructively at the exit of the system giving rise to transmission dips like in a MachZehnder interferometer [59]. Indeed, when the two ribbons are placed symmetrically around the main waveguide, the incident light wave propagates through two identical paths, giving rise to a constructive interference wave at the exit system and therefore a suppression of the transmission zeros as it is illustrated in the Appendix. In Fig. 5(g) and (h), is shown the $H_{z}$-field map at $9.53 \mathrm{THz}$ for the mode (1a), and at about $16.05 \mathrm{THz}$ for the mode (3a). We remark that $H_{z}$ field is strongly localized around the upper ribbon, and almost suppressed around the lower one in both figures; this is even more visible in Fig. 5(h) (the excited mode being more localized around the ribbon in this case). This points to the fact that at the transmission zeros (1a) and (3a) frequencies, the lower and the upper ribbons strongly interfere destructively, resulting in the excitation of the upper ribbon and the almost suppression of the lower one. A similar effect has been observed in [41], where two uncoupled graphene ribbons (set far apart) interfere destructively, giving rise to the same observed effect as here. The authors named the obtained resonance, plasmon induced absorption or PIA. Nevertheless, in Fig. 5(b) one can notice that around modes (1a) and (3a) the absorption does not peak; and rather exhibits a minimum value before peaking around the transmission peaks. However, the reflection spectrum (and contrary to the absorption spectrum) clearly peaks-up around (1a) and (3a), which enables to conclude that the resonances taking place around these transmission zeros correspond to plasmon induced reflection (PIR) and not to PIA. On the other hand, we would like to point out that the absorption peaking around the four transmission peaks in Fig. 5(b) is related to the strong localization at these resonances within and around the effective cavity, giving rise to an enhanced dissipation in the two (coupled) graphene ribbons.

In order to show the tunability of the bandpass filter proposed here, we have calculated the transmission spectrum of the system for various Fermi-energy levels of the effective cavity, that is, for $E_{F 1}=$ $140 \mathrm{meV}, 160 \mathrm{meV}$ and $180 \mathrm{meV}$. The remaining parameters are left unchanged (as previously). The results are plotted in Fig. 6. We remark a steady increase of the four modes resonance frequencies with $E_{F}$, and such behaviour can be easily explained using Eq. (2), giving the resonance wavelength of a single nano-ribbon.

\subsection{Multi-plasmon induced reflection (PIR)}

Now, we turn our attention into studying the PIR-effect. The geometry design giving rise to PIR consists into inserting a cavity-oscillator along the main waveguide and another identical cavity vertically above it as sketched in Fig. 7(a). The effective cavity-oscillator inserted aside of the GBW is placed at a distance defined by the parameter $g^{\prime}$ from the cavity situated along the main waveguide. Specifically, the distance $g^{\prime}$ is measured from the upper ribbon of the cavity placed along the GBW up to the lower ribbon of the cavity situated upwards. The idea behind this geometry design is that the lower resonator interacting directly with the main waveguide plays the role of the bright mode, whilst the upper one behaves as the dark mode. In Fig. 7(b), we present the transmission and reflection spectra of the system depicted in Fig. 7(a) for $g^{\prime}=150 \mathrm{~nm}$, $l=250 \mathrm{~nm}, w=110 \mathrm{~nm}, d=10 \mathrm{~nm}, E_{F 1}=160 \mathrm{meV}$ (for both the lower and upper two CGNRs) and $E_{F 0}=140 \mathrm{meV}$. One notes the

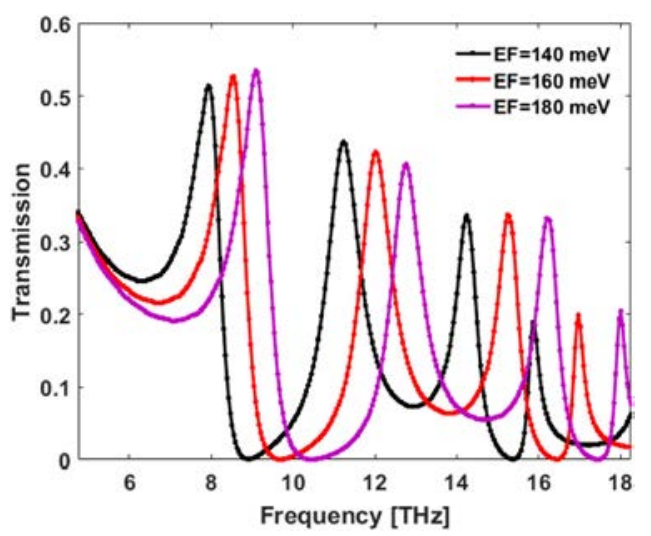

Fig. 6. Transmission spectrum of the structure sketched in Fig. 5(a) for various values of $E_{F 1}$. The remaining parameters are hold constant as: $l=250 \mathrm{~nm}, w=110 \mathrm{~nm}, d=10 \mathrm{~nm}$, and $E_{F 0}=140 \mathrm{meV}$.

splitting-up of the four resonances peaks observed in Fig. 5(b), resulting in eight transmission peaks. The $H_{z}$-field maps at these (eight) peaks frequencies show that they are either symmetric or anti-symmetric, with respect to the mid-plane between the cavities. An example of the $H_{z}$-field maps at the third and fourth peaks frequencies, referred to as (2a) and (2c) in Fig. 7(b), are given in Fig. 7(c) and (d), respectively and can be compared with the field map of mode 2 in Fig. 5(b) from which they originate. Symmetry plane $C_{x}$ (parallel to $x$-axis) lying inbetween the cavities, is highlighted with dotted lines in these figures. It is worth mentioning that the cavities are only evanescently coupled to the GBW, and therefore discussing their modes symmetry with respect to the plane $C_{x}$ is still approximately valid although the GBW breaks this symmetry with respect to $C_{x}$. We remark that the transmission peak (2a) is symmetric, whilst the transmission peak (2c) corresponds to an antisymmetric mode, with respect to $C_{x}$. The observed transmission peaks in Fig. 7 (b) arise because of the coupling of the two identical cavities, inducing the splitting of transmission peaks.

One can notice that transmission dips in-between each couple of the new emerging transmission peaks have shown-up. We refer to these as (1b), (2b), (3b) and (4b) in Fig. 7(b). In particular, the four dips (1b), (2b), (3b) and (4b) arise around the transmission peaks frequencies of Fig. 5(b), that is, around modes (1), (2), (3) and (4) (respectively) of the cavity-oscillator. The $H$ - magnetic field amplitude maps at the dips (1b), (2b), (3b) and (4b) frequencies are presented in Fig. 7(e)-(h), respectively. It can be seen that the upper cavity is rather strongly excited, whilst the lower one is much less excited or almost suppressed. Moreover, the excited modes within the upper cavity are identical to the modes (1), (2), (3) and (4), according to their structures. This indicates that extreme destructive interferences take place at the dips frequencies between the two cavities. The mechanism of these destructive interferences is identical to the one behind the observed transmission zeros labelled (1a) and (3a) in Fig. 5(b). Indeed, an incident SPP guided mode has two different optical paths (through the two effective cavities) which give rise to destructive interferences between the paths at the exit of the cavities leading to the formation of the observed dips. For the same reasons mentioned above (when analysing Fig. 5), the four transmission dips should be considered as PIR-resonances rather than PIA-effects, given that high reflection peaks are generated at these resonances, while the absorption coefficient is not really affected (and does not peak) based on Fig. 7(b). Interestingly, the transmission zeros labelled (1a) and (3a) in Fig. 5(b) are still observed in Fig. 7(b) around almost the same frequencies. These PIR resonances are labelled (1d) and (3d) in Fig. 7(b). These dips are induced by the lower cavity especially the upper ribbon of the cavity placed along the GBW as indicated by the $H_{z}$-field maps (not shown here). Alongside with the multi-PIR effects proposed here based on our effective cavity-oscillator, 
(a)
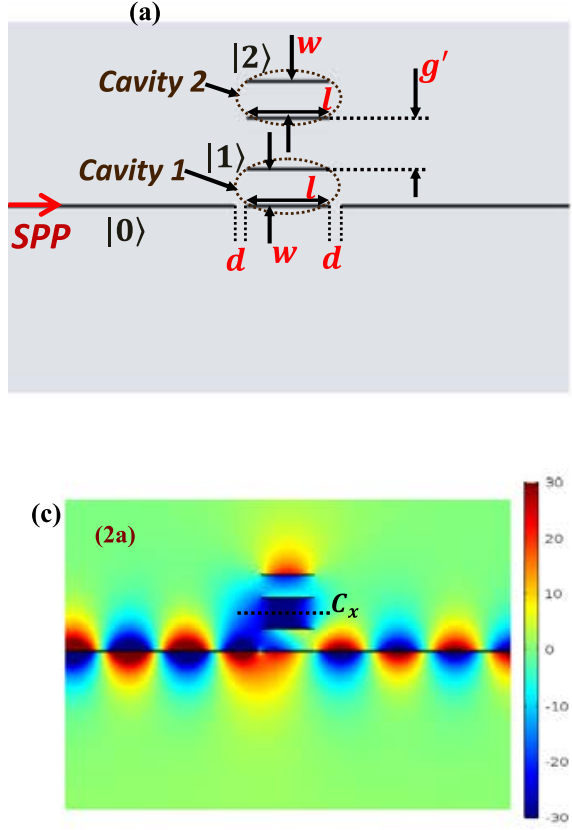

(e)
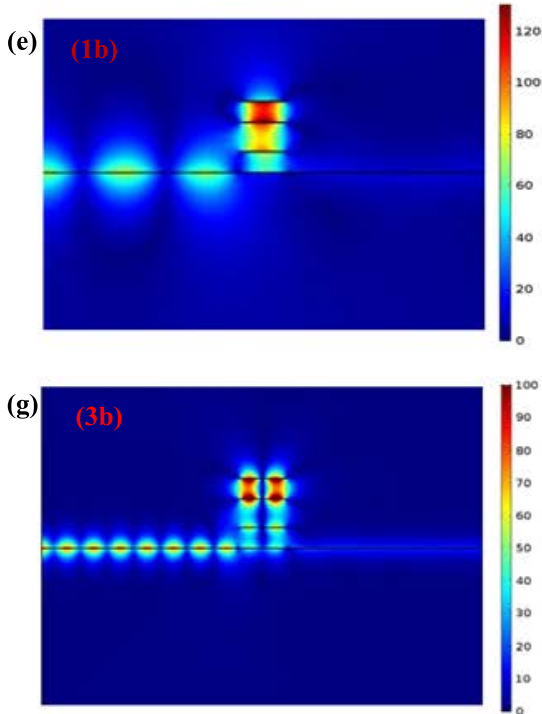

(b)

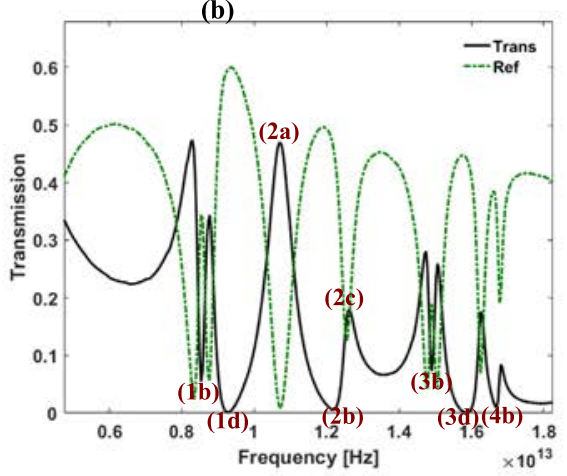

(d)
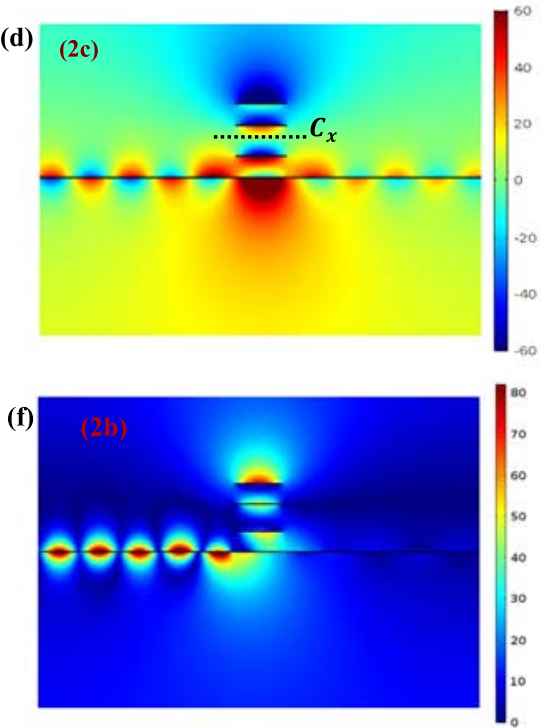

(h)

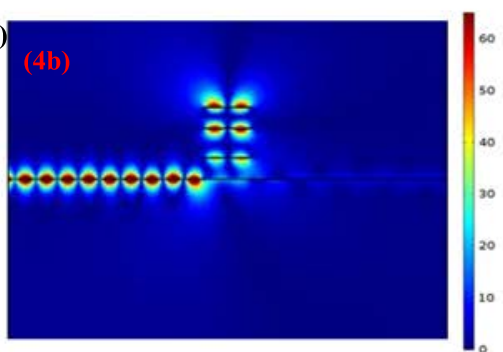

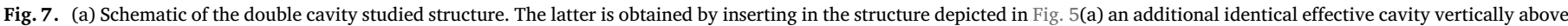

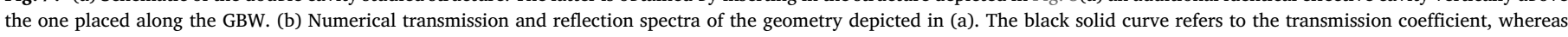

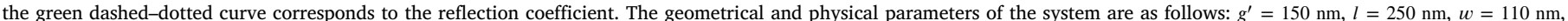

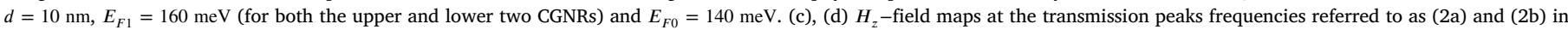

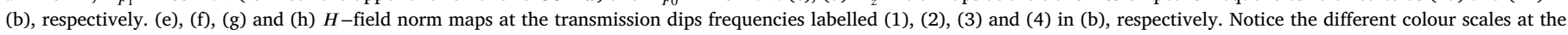
right hand of Figs. (c)-(h).

higher Q-factors PIR-resonances maybe achieved with our structure as in the case of PIR resonances labelled (1b) and (3b) in Fig. 7(b). It is also worthwhile indicating that the adjustment of the multi-PIR-resonances frequencies could be readily achieved by shifting the Fermi energy levels of the uppers and lowers two CGNRs.

The formation and build-up of the (multiple) PIR window is caused by the destructive interferences of the upper and lower cavities, thus, it is quite natural to investigate the effect of the distance $g^{\prime}$ (representing the coupling strength between the two resonators) on the PIRresonances. In other words, $g^{\prime}$ contributes to the optimization of the PIR window. In Fig. 8(a) and (b) are presented the transmission spectra of the studied structure for two different values of $g^{\prime}$, namely $150 \mathrm{~nm}$ and $220 \mathrm{~nm}$.
PIR-resonances referred to as (1) and (3) in Fig. 8(a) are manifestly degraded as $g^{\prime}$ is increased from $150 \mathrm{~nm}$ to $220 \mathrm{~nm}$ (Fig. 8(b)). Specifically, their associated dips get much less deeper and tend to almost disappear. At the same time the FWHM of these dips decreases with the increase of $g^{\prime}$. On the other hand, PIR-resonances labelled (2) and (4) in Fig. 8(a) get sharper with increasing $g^{\prime}$. In particular, PIR-resonance (2) is clearly best defined in Fig. 8(b) when $g^{\prime}=220 \mathrm{~nm}$, as opposed to the case when $g^{\prime}=150 \mathrm{~nm}$. In Fig. 8(c), we present the modulus of the magnetic field at the transmission dip (2) frequency of Fig. 8(b). One notes a stronger excitation of the mode (2) oscillating within the upper cavity as compared to the case when $g^{\prime}=150 \mathrm{~nm}$ (Fig. 7(f)). This is related to a weaker coupling intensity between the lower and upper cavities when $g^{\prime}$ increases. Therefore, the FHWM of the PIR-resonance (2) is decreased or alternatively the associated transmission dip is best 

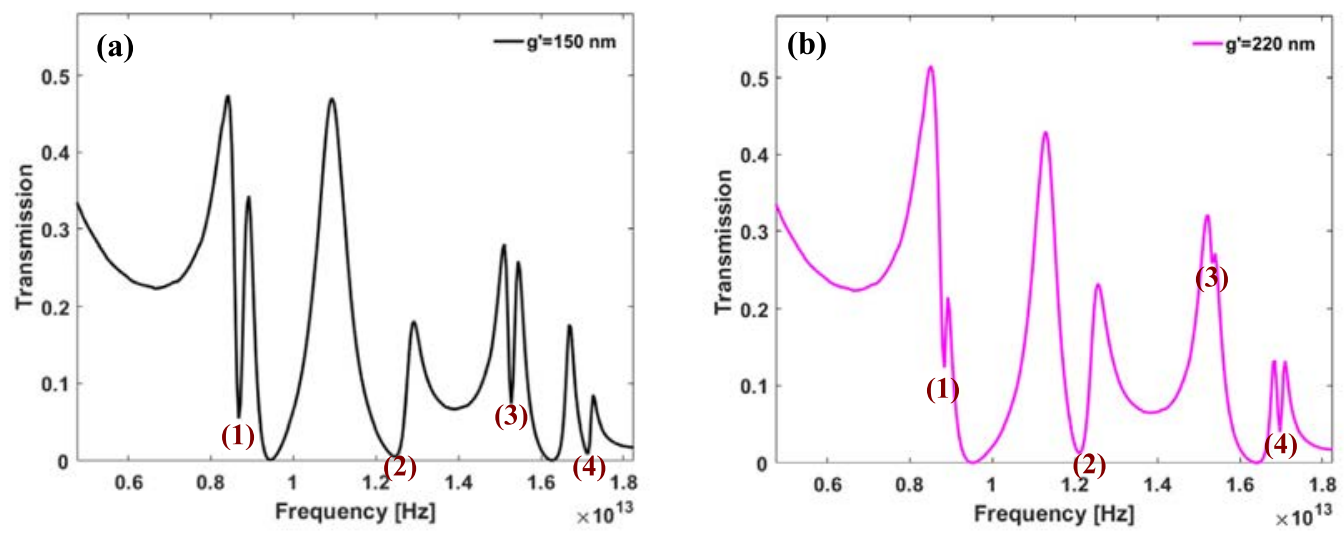

(c)

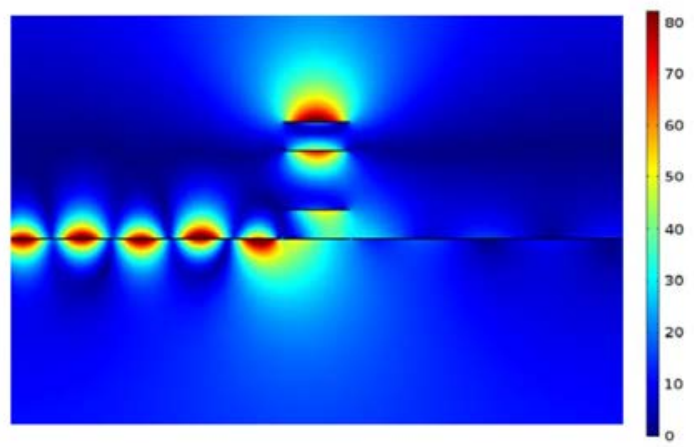

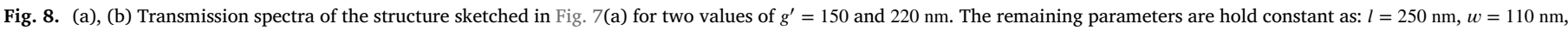

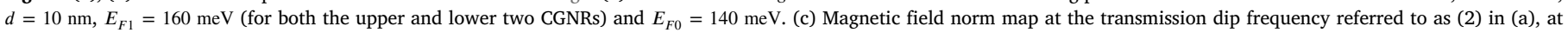
around $f_{r 2}=12.1 \mathrm{THz}$. Notice that the colour scale is identical to the one in Fig. 7(f).

defined, hence, a stronger localization of mode (2) is observed within the upper cavity.

In addition to the amplitude, it is interesting to investigate the phase of the transmission coefficient, $\varphi$, of the proposed structure (Fig. 7(a)). In Fig. 9(a), we have plotted the phase variation as a function of the frequency such that $g^{\prime}=150 \mathrm{~nm}, l=250 \mathrm{~nm}, w=110 \mathrm{~nm}, d=10 \mathrm{~nm}$, and $E_{F 0}=140 \mathrm{meV}$; and as before $E_{F 1}=160 \mathrm{meV}$ is maintained constant for both the upper and lower two CGNRs. The phase drops around the four PIR-resonances labelled (1b), (2b), (3b) and (4b) in Fig. 7(b), but also around PIR resonances labelled (1d) and (3d) in the same figure. Nevertheless, the phase drops are quite small due to the large absorption in the system. The signature of the phase drops is even more visible in Fig. 9(b) where the time delay, defined as $\tau=-\frac{d \varphi}{d \omega}$, is depicted. In particular, negative time delays are observed around each PIR window, or equivalently a time advance around each PIRresonance [60], giving rise to multiple fast-light features in the proposed graphene-based resonator system. Such features could lead to the design of new integrated optical devices and provide a way for the control of a light signal transmission.

\subsection{Multi-plasmon induced transparency (PIT)}

In this section, we show the possibility of realizing multi-plasmon induced transparency (PIT) by slightly changing the configuration set of the structure depicted in Fig. 7(a). Specifically (see Fig. 10(a)), each cavity is now placed aside of the GBW, at a distance $g_{i}$ (where $i=1$ or 2), counting from the lower ribbon (of the cavity) down to the bus waveguide, giving rise to the V-shape state (in analogy with the three level atomic system). In that configuration, both resonators act as the bright mode, due to their direct coupling with the main waveguide. In order to achieve PIT resonances, we slightly detune the Fermi-energy levels of the upper and lower two CGNRs of Fig. 10(a), in order to squeeze-up transmission peaks/bands in between the transmission dips of the detuned cavities. The corresponding transmission spectrum is presented in Fig. 10(b), for $g_{1}=g_{2}=130 \mathrm{~nm}, l=250 \mathrm{~nm}, w=110 \mathrm{~nm}$, $E_{F 0}=140 \mathrm{meV}, E_{F 1}=160 \mathrm{meV}$ and $E_{F 2}=180 \mathrm{meV}$. As a matter of comparison, we also give (in green dashed and red dashed-dotted curves) the transmission spectrum for the upper and lower effective cavities, coupled alone to the GBW. We observe the emergence of four transmission bands surrounded by the transmission dips of each resonator. We refer to the transmission bands as (1), (2), (3) and (4) in Fig. 10(b). In particular, the PIT-transmission peak (1) seems to have a quite low FHWM as compared to the other peaks. The second peak near mode (2) is relatively large, while the transmission bands showing-up in between modes (3) and (4) (of each cavity) are quite large as these modes (associated with each resonator) are further separated in terms of their frequencies. In Fig. 10 (c) and (d), we present the $H_{z}$ - field at the EIT-resonances peaks (1) and (2) frequencies, given that the latters are rather well excited and have a relatively low FHWM. It can be seen based on these figures that both upper and lower cavities are well excited, but clearly in anti-phase. As a consequence, the two cavities acting as radiative (coupled) oscillators interfere destructively, and decouple from the guided SPP mode in the GBW, resulting in its transmission. It is worthwhile reporting that similar multi-PIT peaks have been achieved lately in a graphene-based coupled nanowires structure set in a V-like state [61]. Nonetheless, our proposed design offers more compactness and is probably easier to fabricate.

One of the most important features of an EIT-resonance is the strong dispersion that takes place within the induced transparency window, giving rise to slow light effect. To showcase this phenomenon, we have calculated the time delay $\tau=-\frac{d \varphi}{d \omega}$, first, and then derive the group velocity according to $v_{g}=L / \tau$, where $L$ is the effective distance 
(a)

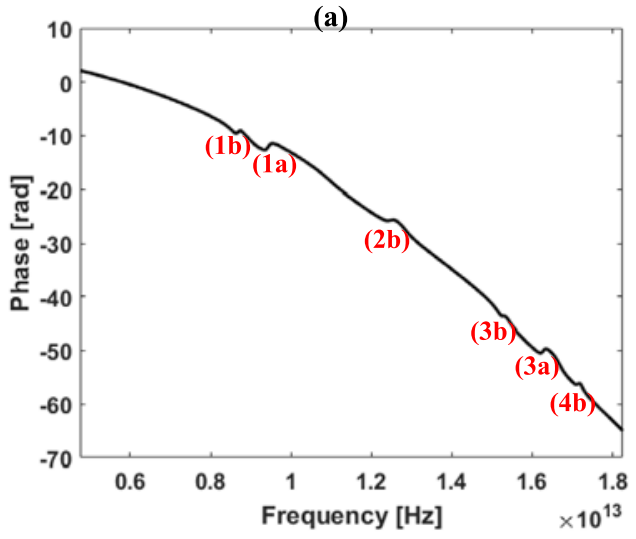

(b)

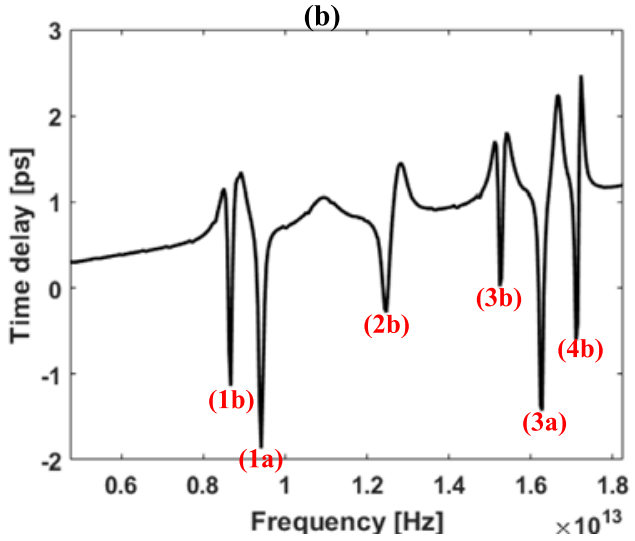

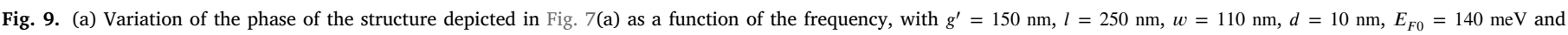
$E_{F 1}=160 \mathrm{meV}$ (for both upper and lower two CGNRs). (b) Same as (a) but for the delay time.

(a)

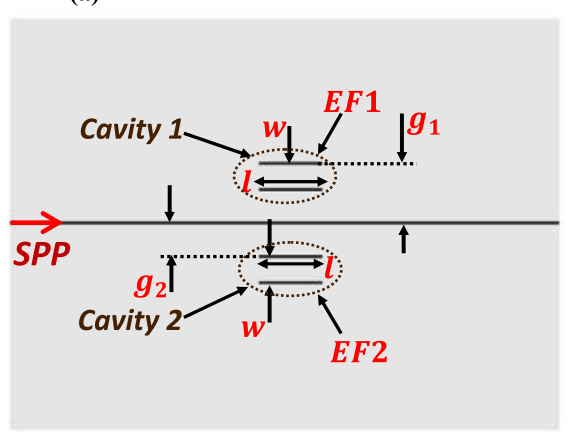

(c)

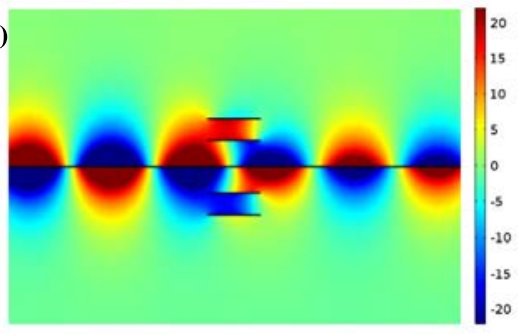

(b)
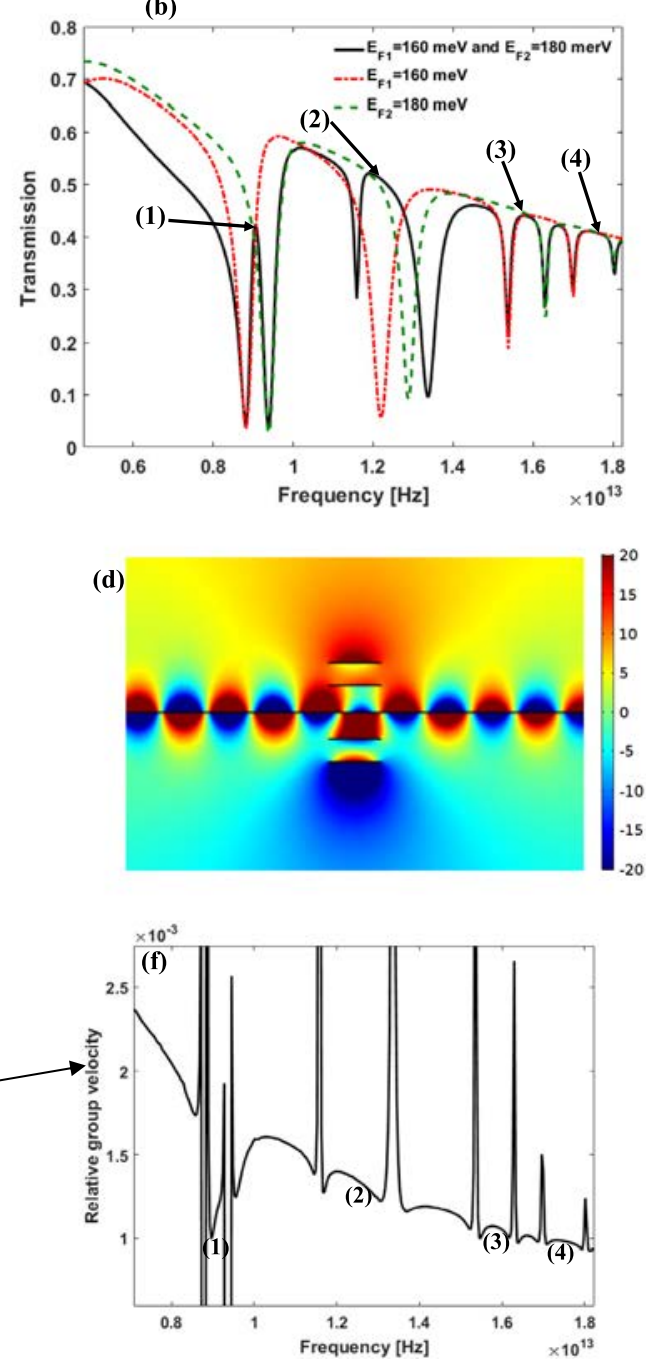

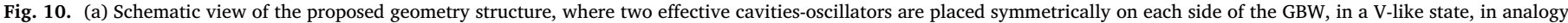

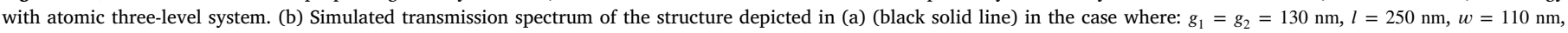

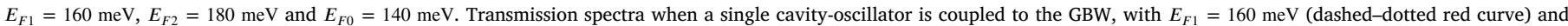

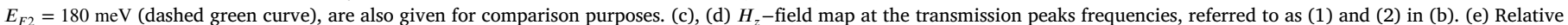
group velocity of the structure depicted in (a). (f) A magnification of (e) is performed around PIT resonances (1), (2), (3) and (4), to better visualize the slow light effect. 
(a)
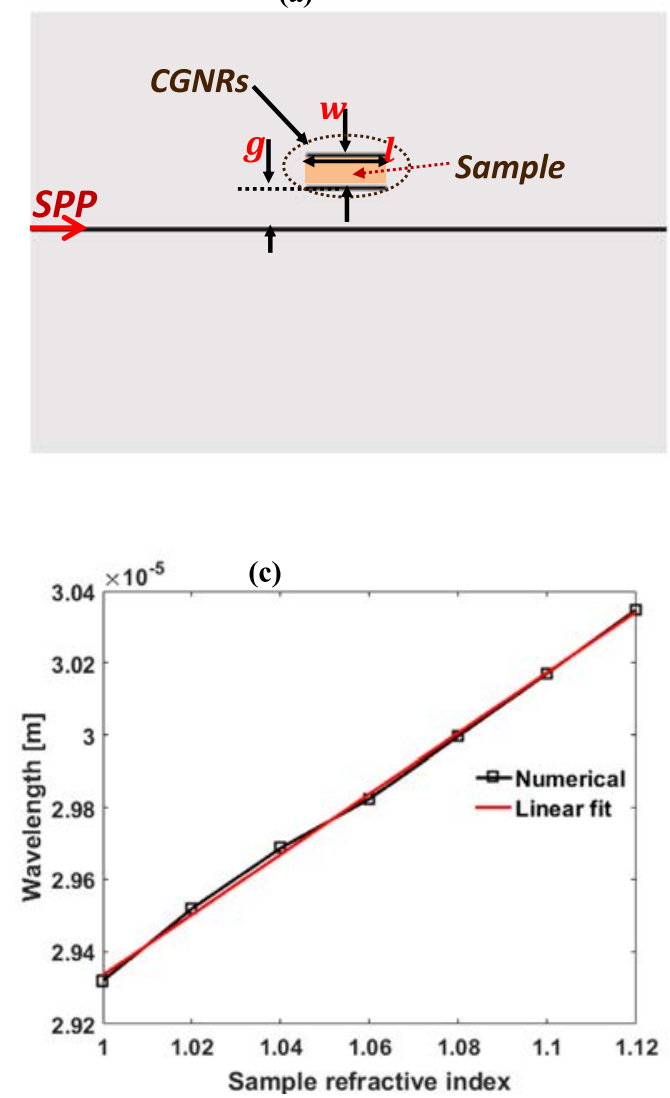

(b)

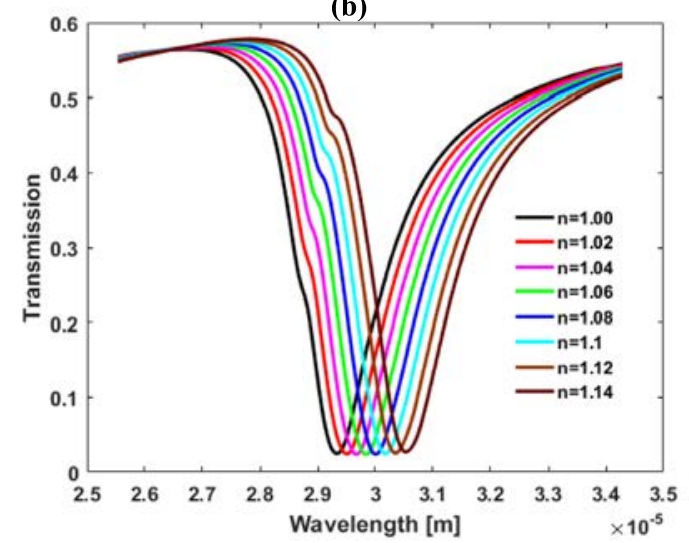

(d)

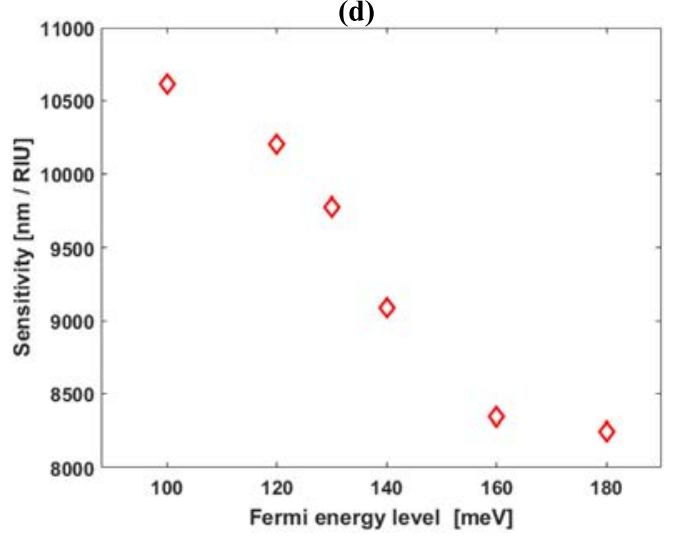

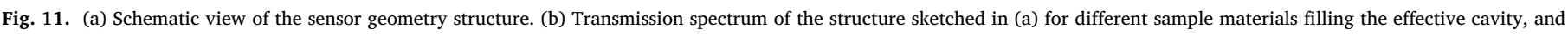

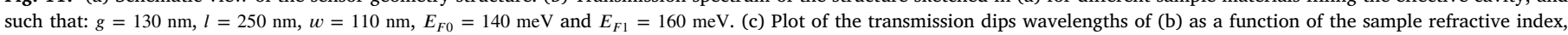

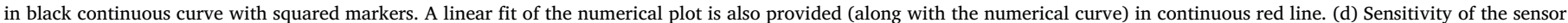
depicted in (a) as a function of the Fermi energy level of the two CGNRs.

travelled by an incident light signal. In practice, L is approximately equal to the length of the structure $2.325 \mu \mathrm{m}$. In Fig. 10(e), is presented the relative group velocity defined as $V_{g}=v_{g} / c$, where $c$ is the speed of light in vacuum. The physical and geometrical parameters are taken such as: $g_{1}=g_{2}=130 \mathrm{~nm}, l=250 \mathrm{~nm}, w=110 \mathrm{~nm}, E_{F 0}=140 \mathrm{meV}$, $E_{F 1}=160 \mathrm{meV}$ and $E_{F 2}=180 \mathrm{meV}$. A zoom-in around the PITtransmission bands (highlighted by (1), (2), (3) and (4) in Fig. 10(e)) is performed in Fig. 10(f) to better visualize the slow-light features. As expected, the relative group velocity turns negative around the dips frequencies and falls down (subluminal effect) as expected meaningfully within the transparency windows: (1), (2), (3) and (4). The effect is more visible in Fig. 10(f). This is particularly true around the PIT-resonance (1), where the time delay associated with this peak is such that $\tau \sim 1 / \Delta \omega$, $\Delta \omega$ being smaller for peak (1) in comparison with the rest of the peaks. As a result, the time delay is bigger for peak (1) and, in turns $v_{g} \sim / \tau$ is slower.

\subsection{Nanosensor}

The showcased effective cavity-oscillator in this work can be harnessed to demonstrate the possibility of realizing an ultra-compact refractive index nano-sensor. The geometry design of the device consists in the graphene bus waveguide side-coupled to the effective resonator, filled with the sample under investigation. Fig. 11(a), presents a schematic view of the structure. The sample (gas or liquid) to be detected is depicted in a shaded pink colour. Let us mention that the cavity-oscillator can also be placed along the GBW (like in Fig. 5(a)), to realize the sensor. Nonetheless, the associated transmission dips to the cavity modes are sharper when the cavity is side-coupled to the
GBW, due to indirect coupling with the GBW in this case, which might result in a better sensitivity of the device. Numerical simulations point towards the low and high frequency modes (modes (1) and (4) resp.) of the cavity (studied in Fig. 2) as being the most sensitive to a refractive index change. This could be related to the modes structure/nature in comparison with the other modes. Nevertheless, mode (4) is quite weakly excited as opposed to mode (1). As a result, we will focus herein on mode (1) in the aim of analysing the performances of the sensor. To do this, the material sample inside the cavity-oscillator and its refractive index are changed; and we then investigate the resonance wavelength variations of mode (1) as a function of the sample refractive index changes. In practice, the measure of the resonance wavelength enables the determination of the unknown material, by comparing it with a look up table [62]. Quantitatively, the performance of the proposed sensor is determined by calculating its sensitivity, defined as the variation of the resonance wavelength with respect to the resonator refractive index, that is, $S=d \lambda / d n$ ( $n$ is the sample refractive index); where $S$ is given in nanometre per refractive index units $\mathrm{nm} / \mathrm{RIU}$.

Fig. 11(b) gives the transmission spectrum of the system versus wavelength focused around mode (1) for different values of the sample refractive index starting out from $n_{s}=1$. The parameters are: $g=$ $130 \mathrm{~nm}, l=250 \mathrm{~nm}, w=110 \mathrm{~nm}$ and $E_{F 1}=160 \mathrm{meV}$. We note an important sensitivity of the device as the SPP mode resonance wavelength $\lambda_{r}$ is noticeably shifted when the refractive index of the sample changes by only $2 \%$. To be more quantitative, we have plotted the resonance wavelength $\lambda_{r}$ against $n_{s}$, and performed a linear fit of the numerical data; the results are presented in Fig. 11(c). Based on this figure, we evaluate the sensitivity of the proposed sensor as $S=8407 \mathrm{~nm} / \mathrm{RIU}$. Recently, a sensitivity of $7500 \mathrm{~nm} /$ RIU has been achieved in [62], whilst 
(a)
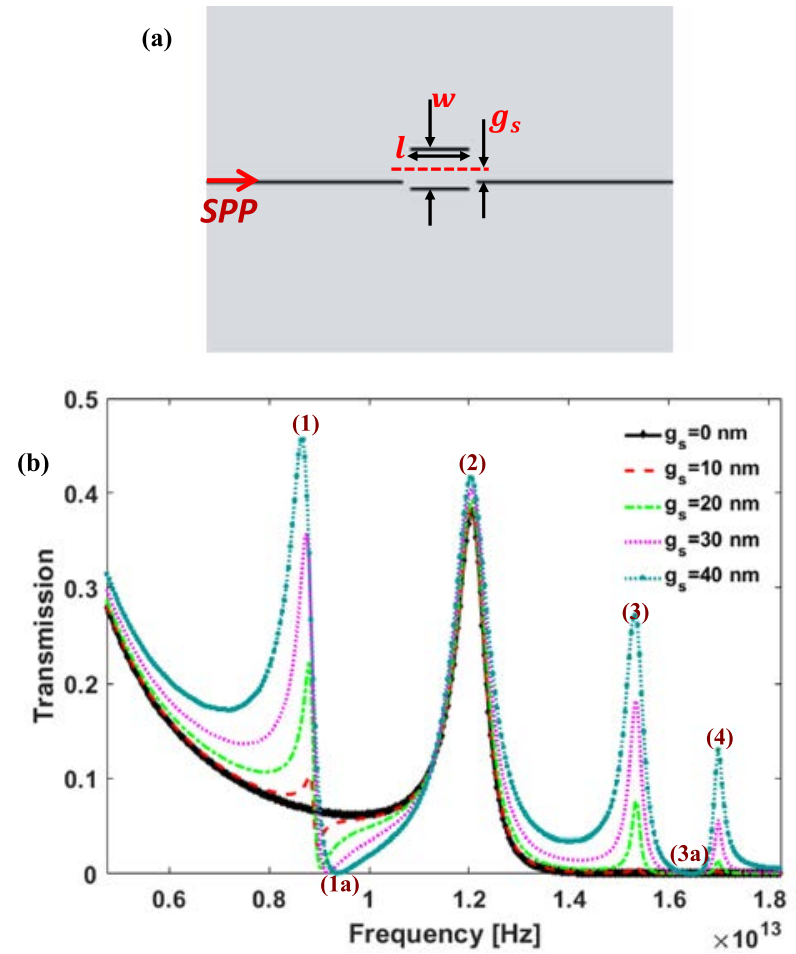

Fig. 12. (a) Sketch of the structure depicted in Fig. 5(a) such that the two CGNRs forming the cavity are asymmetrically set with respect to the main waveguide. The asymmetry is defined by the parameter $g_{s}$, amounting to the symmetry plane of the cavity vertical displacement upwards (with respect to the main waveguide). (b) Corresponding transmission spectrum for different values of $g_{s}$, in the case when $l=250 \mathrm{~nm}, w=110 \mathrm{~nm}$, $E_{F 1}=160 \mathrm{meV}, E_{F 0}=140 \mathrm{meV}$ and $d=10 \mathrm{~nm}$.

in [63] a value of only $S=4800 \mathrm{~nm} / \mathrm{RIU}$ was reached. It is worthwhile mentioning that in these works more sophisticated graphene based resonators was studied such as circular graphene nanowires, whereas our geometry design (while being slightly more sensitive) is quite simple in comparison. The resonance wavelength around which the sensor does operate (mode (1) wavelength) may be readily tuned by adjusting the Fermi energy level. It is of interest then to investigate how the sensitivity of the sensor does evolve with the parameter $E_{F 1}$ (Fermi energy level of the two CGNRs). The corresponding data is given in Fig. 11(d), where we have plotted the sensitivity of the sensor $(S=d \lambda / d n)$ as a function of the Fermi energy level, $E_{F 1}$. Clearly, the sensitivity of the sensor increases when the Fermi energy level of the effective cavity decreases. In particular, the sensitivity reaches an astonishingly higher value of $10482 \mathrm{~nm} / \mathrm{RIU}$ for $E_{F 1}=100 \mathrm{meV}$. Let us mention that a similar trend is observed in [62].

As a conclusion, the simplicity of the geometry design proposed in this work, makes it ideal for the fabrication of ultra-compact and highly sensitive new generation of graphene-based nanosensors.

\section{Conclusion}

In this work, we investigated theoretically and numerically a novel ultra-compact graphene-based structure for the realization of multiplasmon induced reflection, transmission and a highly sensitive nanosensor. The base structure consists in a bus waveguide made out of graphene, and two coupled graphene nano-ribbons, acting as a molecule-like system or an effective (rectangular) cavity-like entity. The proposed system operates in the terahertz regime. The two CGNRs and the waveguide are embedded in a dielectric material. The eigen modes of the two CGNRs arise from the hybridization of the SPP modes of the single ribbons, due to strong near field interaction. In particular, the effective cavity-oscillator has four eigen-modes in the frequency range of interest. Based on temporal coupled mode theory, a fitting of the numerically determined cavity eigen-modes was provided. Before studying multi-PIR/PIT effects, we have investigated the configurations sets where the effective cavity is whether placed aside or along the main waveguide, giving rise to multiple-rejection or selection filtering, respectively. The insertion of two identical cavities along and above the main waveguide generates four transmission dips within the transmission spectrum of the system. These multiple dips are shown to correspond to plasmon induced reflection (PIR) phenomenon. The origin of the resonances is explained by extreme destructive interferences taking place between the cavities at the PIR resonances frequencies. Fast light features around the PIR-resonances are highlighted. A slight change in the two cavities configuration set within the system is shown to give rise to multiple-plasmon induced transparency (PIT)-transmission peaks. This is achieved by placing each cavity-oscillator symmetrically on each side of the main waveguide, that is, in a V-like state (in analogy with three level atomic systems). The physical mechanism is explained as follows: by slightly detuning one of the two cavities properties (Fermi energy level in this work), one can squeeze-up a PIT-resonance between the transmission dips of each cavity. Slow light effect around each PIT resonance is showcased. Finally, we demonstrated the possibility of realizing graphene-based highly sensitive refractive index change nanosensor. The device is composed out of the bus waveguide sidecoupled to the effective cavity-oscillator. The latter is filled out with the sample (liquid or gas) to be detected. The sensitivity of the structure is shown to be strongly dependent on the Fermi energy level of the ribbons forming the cavity, which opens up the possibility of dynamically tuning the sensor sensitivity. The proposed sensor offers the advantage of being quite simple as opposed to the showcased graphene-based sensors in the literature. The versatility and simplicity of the proposed structure could help the design of highly integrated optical devices such as nanoscale optical filters, highly sensitive nano-sensors and high-speed switches.

Appendix. Origin of the transmission zeros in the double ribbon structure along the waveguide

In order to show the origin of the transmission zeros in the double ribbon structure along the waveguide in Fig. 5(a), we have considered the two ribbons forming the effective cavity slightly asymmetrically placed with respect to the main waveguide. More specifically, the symmetry mid plane $S_{y}$ (highlighted with a dashed line in Fig. 12(a)) of the cavity is vertically shifted upwards by an amount labelled $g_{s}$ with respect to the main waveguide. Our goal is to show the effect of the position of the cavity along the vertical direction on the transmission spectra along the horizontal waveguide direction.

Fig. 12(b) gives the transmission spectrum of the structure depicted in Fig. 12(a) for different values of $g_{s}$. The remaining parameters are chosen such as $l=250 \mathrm{~nm}, w=110 \mathrm{~nm}, E_{F 1}=160 \mathrm{meV}, E_{F 0}=140 \mathrm{meV}$ and $d=10 \mathrm{~nm}$. The width separation between the two ribbons is $w=110 \mathrm{~nm}$. The value of $g_{s}=0$ corresponds to symmetric ribbons (cavity) with the waveguide axis, whereas $g_{s} \neq 0$ represents asymmetric cavity. Starting from $g_{s}=40 \mathrm{~nm}$, one can notice the existence of four peaks labelled (1), (2), (3) and (4) (cyan curve). The peaks (1) and (2) as well as (3) and (4) are separated by well defined zeros labelled 1(a) and (3a) respectively. The first peak (1) exhibits a Fano-like shape, its amplitude and width increase when $g_{s}$ increases. Also, we note that the position of this peak shifts slightly to higher frequencies and at the same time the first transmission zero goes towards lower frequencies (see for example the red curve), with the decrease of $g_{s}$. For $g_{s}=0 \mathrm{~nm}$, both peak (1) and dip (1a) merge together around 9THz giving rise to non-zero transmission (black curve). This result can be understood as a result of the interference between the waves propagating through two pathways (i.e., ribbons). When, the ribbons are set asymmetrically along the main waveguide, we assist to destructive interference of the two waves and therefore the existence of the transmission zero, this is similar to a Mach-Zehnder interferometer. Whereas in the case of symmetric 
ribbons, the waves interfere constructively at the exit of the system and consequently the transmission does not vanish. Similar results have been found in asymmetric and symmetric waveguide loops [59]. The mode labelled (2) remains robust to the variation of $g_{s}$, its shape changes slightly. As concerns the modes labelled (3) and (4), their transmission spectra present a different behaviour than peaks (1) and (2). In particular, we can notice that their intensities decrease and almost vanish when $g_{s}$ goes to zero, especially for the higher mode (4). However, the transmission zero (3a) disappears for $g_{s}=0 \mathrm{~nm}$, this is not clearly apparent at the scale of Fig. 12(b). Here we would like to notice an interesting behaviour, although out of the scope of this work, namely the existence of a well-defined resonance filtering while the transmission is almost suppressed over a broad frequency range. It is worth mentioning that the amplitudes of modes (3) and (4) are very sensitive to the width $w$ separating the two ribbons, especially both ribbons interact strongly with the incident wave in the waveguide for lower values of $w$ at these modes frequencies. For instance, we have checked that for $w=60 \mathrm{~nm}$ and $g_{s}=15 \mathrm{~nm}$, the peaks (3) and (4) reach almost $23 \%$ and $20 \%$ respectively; these peaks are separated by a well-defined transmission zero. However, when $g_{s}$ goes to zero, the peak (3) merges with the transmission zero (3a) giving rise to non-zero transmission value and only the peak (4) continue to exist.

\section{References}

[1] M. Fleischhauer, A. Imamoglu, J.P. Marangos, Rev. Modern Phys. 77 (2005) 633641.

[2] J. Zhang, G. Hernandez, Y. Zhu, Opt. Lett. 33 (2008) 46-3.

[3] G. Heinze, C. Hubrich, T. Halfmann, Phys. Rev. Lett. 111 (2013) 033601-033605.

[4] M.M. Alotaibi Hessa, B.C. Sanders, Phys. Rev. A. 94 (2016) 053832-0538311.

[5] N. Papasimakis, V.A. Fedotov, N.I. Zheludev, S.L. Prosvirnin, Phys. Rev. Lett. 101 (2008) 253903-253904

[6] M.F. Yanik, W. Suh, Z. Wang, S. Fan, Phys. Rev. Lett. 93 (2004) 233903.

[7] Q. Xu, S. Sandhu, M.L. Povinelli, J. Shakya, S. Fan, M. Lipson, Phys. Rev. Lett. 96 (2006) 123901

[8] M. Wen, L. Wang, X. Zhai, Q. Lin, S. Xia, Europhys. Lett. 116 (2017) 44004.

[9] S.X. Xia, X. Zhai, L.L. Wang, B. Sun, J.Q. Liu, S.C. Wen, Opt. Express 24 (2016) $17886-17899$.

[10] W.L. Barnes, A. Dereux, T.W. Ebbesen, Nature 424 (2003) 824-830.

[11] Z. Han, S.I. Bozhevolnyi, Opt. Express 19 (2011) 3251-3257.

[12] S.A. Mikhailov, K. Ziegler, Phys. Rev. Lett. 99 (2007) 016803

[13] W. Gao, J. Shu, C. Qiu, Q. Xu, ACS Nano. 6 (2012) 7806-7813.

[14] A.K. Geim, K.S. Novoselov, Nat. Matter. 6 (2007) 183-191.

[15] Z. Su, X. Chen, J. Yin, X. Zhao, Opt. Lett. 41 (2016) 3799-3802.

[16] L. Luo, K. Wang, K. Guo, F. Shen, X. Zhang, Z. Yin, Z. Guo, J. Opt. 19 (2017) 104169.

[17] A.Y. Nikitin, F. Guinea, F.J. Garcia-Vidal, L. Martin-Moreno, Phys. Rev. B 84 (2011) 161407

[18] X. Zhu, W. Yan, N.A. Mortensen, S. Xiao, Opt. Express 21 (2013) 3486-3491.

[19] J.S. Gómez-Díaz, J. Perruisseau-Carrier, Opt. Express 21 (2013) 15490-15504.

[20] Z. Qi, Z. Zhu, W. Xu, J. Zhang, C. Guo, K. Liu, X. Yuan, S. Qin, J. Opt. 18 (2016) 065003.

[21] Z. Fang, Z. Liu, Y. Wang, P.M. Ajayan, P. Nordlander, N.J. Halas, Nano Lett. 12 (2012) 3808-3813,

[22] Z. Fang, Y. Wang, A.E. Schlather, Z. Liu, P.M. Ajayan, F.J. García de Abajo, P. Nordlander, X. Zhu, N.J. Halas, Nano Lett. 14 (2013) 299-304.

[23] Z. Huang, L. Wang, B. Sun, M. He, J. Liu, H. Li, X. Zhai, J. Opt. 16 (2014) 105004.

[24] S. Asgari, N. Granpayeh, J. Nanophoton. 11 (2017) 026012.
[25] F. Xing, Z.B. Liu, Z.C. Deng, X.T. Kong, X.Q. Yan, X.D. Chen, Q. Ye, C.P. Zhang, Y.S. Chen, J.G. Tian, Sci. Rep. 2 (2012) 908.

[26] M. Pan, Z. Liang, Y. Wang, Y. Chen, Sci. Rep. 6 (2016) 29984.

[27] H. Meng-Dong, K.J. Wang, L. Wang, J.B. Li, J.Q. Liu, Z.R. Huang, Li. Wang, L. Wang, W.D. Hu, X. Chen, Appl. Phys. Lett. 105 (2014) 081903.

[28] C. Zeng, J. Guo, X. Liu, Appl. Phys. Lett. 105 (2014) 121103.

[29] L. Wang, W. Li, X. Jiang, Opt. Lett. 40 (2015) 2325-2328.

[30] X. Han, T. Wang, X. Li, S. Xiao, Y. Zhu, Opt. Express 23 (2015) 31945-31955.

[31] Q. Lin, X. Zhai, L. Wang, B. Wang, G. Liu, S. Xia, Europhys. Lett. 111 (2015) 34004.

[32] H.J. Li, X. Zhai, L. L.Wang, Appl, Phys. Express. 8 (2015) 092201.

[33] S.F. Mingaleev, A.E. Miroshnichenko, Y.S. Kivshar, Opt. Express 16 (2008) 11647.

[34] Z. Vafapour, A. Zakery, Plasmonics 11 (2016) 609-618.

[35] J.X. Jiang, Q.F. Zhang, Q.X. Ma, S.T. Yan, F.M. Wu, X.J. He, Opt. Mater. Express. 5 (2015) 1962-1971

[36] X. He, Y. Yao, Y. Huang, Q. Zhang, L. Zhu, F. Wu, G. Ying, J. Jiang, Opt. Commun. 407 (2018) 386-391.

[37] D. Chun-Feng, Z. Ya-Ting, Y. Jian-Quan, S. Chong-Ling, X.D. Gang, Z. Gui-Zhong, Chin. Phys. B. 23 (2014) 124203.

[38] Y. Liu, Y.O. Zhang, X.R. Jin, S. Zhang, Y.P. Lee, Opt. Commun. 371 (2016) 173-177.

[39] N. Liu, T. Weiss, M. Mesch, L. Langguth, U. Eigenthaler, M. Hirscher, C. Sonnichsen, H. Giessen, Nano. Lett. 10 (2010) 1103-1107.

[40] P.N. Huang, S.-X. Xia, G.-L. Fu, M.-Z. Liang, M. Qin, X. Zhai, L.-L. Wang, Opt. Commun. 410 (2018) 148-152.

[41] M. Wen, L. Wang, X. Zhai, Q. Lin, S. Xia, EPL. 116 (2016) 44004.

[42] T. Zhang, J. Zhou, J. Dai, Y. Dai, X. Han, J. Li, F. Yin, Y. Zhou, K. Xu, J. Phys. D: Appl. Phys. 51 (2018) 055103.

[43] H.-J. Li, L.-L. Wang, X. Zhai, IEEE Photonics Technol. Lett. 28 (2016) 1454.

[44] Q. Lin, X. Zhai, L.-L. Wang, X. Luo, G.-D. Liu, J.-P. Liu, S.-X. Xia, Appl. Phys. Express. 9 (2016) 062002.

[45] J. Chen, M. Badioli, P. Alonso-González, S. Thongrattanasiri, F. Huth, J. Osmond, M. Spasenović, A. Centeno, A. Pesquera, P. Godignon, A.Z. Elorza, N. Camara, F.J. García de Abajo, R. Hillenbrand, F.H. Koppens, Nature 487 (2012) 77-81.

[46] Y. Francescato, V. Giannini, S.A. Maier, New J. Phys. 15 (6) (2013) 063020.

[47] S. Asgari, N. Granpayeh, Opt. Commun. 393 (2017) 5-10.

[48] W. Gao, J. Shu, C. Qiu, Q. Xu, ACS Nano 6 (9) (2012) 7806-7813.

[49] Y. Wu, M. Qu, Y. Liu, Z. Ghassemlooy, Plasmonics 12 (2016) 1487-1492.

[50] S.X. Xia, X. Zhai, L.L. Wang, Q. Lin, S.C. Wen, Opt. Express 24 (2016) 427-436.

[51] L. Wang, W. Li, X. Jiang, Opt. Lett. 40 (2012) 2325-2328.

[52] P. Nordlander, C. Oubre, E. Prodan, K. Li, M.I. Stockman, Nano Lett. 4 (2004) 899903.

[53] Y. Pennec, B. Djafari-Rouhani, A. Akjouj, J.O. Vasseur, L. Dobrzynski, J.P. Vilcot, M. Beaugeois, M. Bouazaoui, R. Fikri, J.P. Vigneron, Appl. Phys. Lett. 89 (2006) 101113.

[54] C. Hu, L. Wang, Q. Lin, X. Zhai, T. Han, J. Du, Appl. Phys. Express 9 (2016) 052001.

[55] Q. Lin, X. Zhai, L. Wang, B. Wang, G. Liu, S. Xia, Europhys. Lett. 111 (2015) 3400434006.

[56] R.D. Kekatpure, E.S. Barnard, W. Cai, M.L. Brongersma, Phys. Rev. Lett. 104 (2010) 243902.

[57] M.L. Ladron de Guevara, F. Claro, Pedro A. Orellana, Phys. Rev. B 67 (2003) 195335.

[58] A. Noual, Y. Pennec, A. Akjouj, B. Djafari-Rouhani, L. Dobrzynski, J. Phys.: Condens. Matter 21 (2009) 375301-375306.

[59] E.H. El Boudouti, N. Fettouhi, A. Akjouj, B. Djafari-Rouhani, A. Mir, J.O. Vasseur, L. Dobrzynski, J. Zemmouri, J. Appl. Phys. 95 (2004) 1102-1113.

[60] J.E. Heebner, R.W. Boyd, J. Modern Opt. 49 (2002) 2629.

[61] B. Wei, S. Jian, Opt. Commun. 402 (2017) 66-72.

[62] S. Asgari, Z.G. Kashani, N. Granpayeh, J. Opt. 20 (2018) 045001.

[63] S. Asgari, A. Dolatabady, N. Granpayeh, Opt. Eng. 56 (2017) 067102. 